\title{
Hybrid Electromagnetism-Like Algorithm for Dynamic Supply Chain Network Design under Traffic Congestion and Uncertainty
}

\author{
Javid Jouzdani and Mohammad Fathian \\ School of Industrial Engineering, Iran University of Science and Technology, Narmak, Tehran 1684613114, Iran \\ Correspondence should be addressed to Javid Jouzdani; javidjouzdani@iust.ac.ir
}

Received 24 March 2015; Revised 18 May 2015; Accepted 24 May 2015

Academic Editor: Erik Cuevas

Copyright ( $\odot 2016$ J. Jouzdani and M. Fathian. This is an open access article distributed under the Creative Commons Attribution License, which permits unrestricted use, distribution, and reproduction in any medium, provided the original work is properly cited.

\begin{abstract}
With the constantly increasing pressure of the competitive environment, supply chain (SC) decision makers are forced to consider several aspects of business climate. More specifically, they should take into account the endogenous features (e.g., available means of transportation, and the variety of products) and exogenous criteria (e.g., the environmental uncertainty, and transportation system conditions). In this paper, a mixed integer nonlinear programming (MINLP) model for dynamic design of a supply chain network is proposed. In this model, multiple products and multiple transportation modes, the time value of money, traffic congestion, and both supply-side and demand-side uncertainties are considered. Due to the complexity of such models, conventional solution methods are not applicable; therefore, two hybrid Electromagnetism-Like Algorithms (EMA) are designed and discussed for tackling the problem. The numerical results show the applicability of the proposed model and the capabilities of the solution approaches to the MINLP problem.
\end{abstract}

\section{Introduction}

Since its introduction by [1], Supply Chain Management (SCM) has been a point of interest to both researchers and practitioners. The reason might be the fact that a significant portion of budget and time in companies is spent on supply chain activities [2]. Obviously, the business units involved in these activities operate efficiently if only the whole supply chain network (SCN) is well structured [3]. Therefore, supply chain network design (SCND), as one of the most important strategic decisions, is known to be vital to survival of companies in today's highly competitive business environment. Generally, SCND includes the determination of the location, capacity, number and the technology of the facilities, and the quantities of products transported from one element to another in order to fulfill the demand in each node of SCN [4]. However, several other factors may affect the decisions regarding the design of the SC. In this paper, the multiperiod design of a SC with multiple transportation modes and multiple products considering the time value of money, traffic congestion, and uncertainty in both demand and supply sides is investigated. In what follows the significance of these features in SCND problem is discussed.

In today's business environment, SCs are urged to be more responsible for their business activities regarding the environment and the society. The concern about these aspects of SC activities has led to the introduction of the "Sustainable Supply Chain Management (SSCM)" concepts. Despite the importance of sustainability in SCM, the relevant literature is still rare [5]. SSCM is defined as the integration of the SC's objective considering economic, social, and environmental aspects of decisions in order to enable the long-term operation of the SC [6]. The research on sustainable supply chain network design (SSCND) under uncertainty is gaining ground in recent years. Considering traffic congestion, Jouzdani et al. [7] proposed a model for SCND under demand uncertainty and presented a dairy industry case study. Pishvaee et al. [5] proposed an accelerated Benders decomposition algorithm for sustainable supply chain network design under uncertainty and addressed a case of medical needle 
and syringe supply chain. They consider economic, environmental, and social objectives. Subulan et al. [8] presented a closed-loop supply chain network design for lead/acid batteries. They considered the risk of end-of-life product collection besides other aspects of SCND decision making. Sadjadi et al. [9] proposed a capacitated multiechelon, multiproduct reverse logistic network design with fuzzy demand for endof-life products. Osmani and Zhang [10] presented a sustainable dual feedstock lignocellulosic-based bioethanol SCND model considering uncertainty in demand, supply, and prices of products. A robust closed-loop SCND (CLSCND) model under uncertainty for a case of medical device industry was investigated by [11]. Dubey and Gunasekaran [12] proposed a SSCND model and addressed a case of an Indian Company. In a recent research, Boukherroub et al. [13] proposed and integrated approach to sustainable supply chain planning. A goal programming approach was proposed by [14] for low carbon supply chain configuration for a new product. Environmental impact of supply chain and its total variable and fixed costs are considered as two objectives by Govindan et al. [15] for integrating sustainable order allocation and sustainable supply chain network strategic design with stochastic demand. They proposed a robust hybrid multiobjective metaheuristic comprised of Electromagnetism-Like Algorithm (EMA) and Variable Neighborhood Search (VNS) as a solution approach to their model. For a review of recent studies in the optimization approaches in SSCND, one may refer to a review by Eskandarpour et al. [16]. The subject of traffic congestion in SCND problem has already attracted researchers in the past few years $[7,17]$. Specifically, since roads are of the most widely used transportation systems in many SCs especially for distribution of good in urban areas, the mutual effect between the transportation activities of a SC and the traffic congestion is a significant issue in SSCND. More specifically, in a SC, different vehicles have different effects on traffic congestion and, therefore, traffic congestion should also be considered in decision regarding the amounts of different products transported by different vehicles. As an example, heavy duty vehicles (e.g., trucks) are capable of transporting higher volumes of goods compared to light vehicles (e.g., pickups); however, the impact of heavy duty vehicles on traffic congestion is larger than that of light types. Therefore, considering different transportation modes, their capacities, and their impact on traffic congestion are significant issues in SCND.

The effectiveness of the SCs is significantly affected by the uncertainty rooted in their complex and dynamic nature [18]. Specifically, the fluctuations and uncertainties in demand-side and supply-side in a SC are results of its inherent complex and variable characteristics. In order to capture the uncertainty in the SCs, some authors have proposed Stochastic Programming (SP) models [1921]. However, it is not always possible to precisely determine the distribution parameters [22]. In addition, the unavailability of historical data is a major drawback of SP methods [5]. Therefore, many authors have utilized Possibilistic Programming (PP) $[5,7,23]$ and Scenariobased/Robust Optimization (RO) [24-28] for modeling the epistemic uncertainty in SCND. The reader is referred to the reviews in SCND modeling uncertainty for further reading $[18,29]$.

SCND has its roots in Facility Location Problem (FLP) and inherits its complexity [30]. Therefore, especially in largescale instances of the problem, conventional solution methods fail to provide a solution in a reasonable amount of time. The problem becomes even more complex when several decision factors are involved. The complexity of the large-scale SCND problem has inspired researchers to propose heuristic and metaheuristic solution methods [22]. A hybrid of Particle Swarm Optimization (PSO) and Genetic Algorithm (GA) was proposed by Soleimani and Kannan [31] for tackling a CLSCND problem. Mousavi et al. [32] proposed a modified PSO algorithm for solving an integrated location and inventory control model of a two-echelon supply chain network. Roghanian and Pazhoheshfar [33] utilized a prioritybased GA to solve a reverse logistics network design under uncertainty. A hybrid of Multiobjective Adaptive Memory Programming (MOAMP) and Tabu Search (TS) algorithms was proposed by Cardona-Valdés et al. [34] for a biobjective SCND stochastic problem. The authors [11] applied a hybrid of Memetic Algorithm (MA) and Variable Neighborhood Search (VNS) to solve their robust model for a closed-loop global SCND under uncertainty. A nondominated sorting genetic algorithm (NSGA-II) was utilized by Pasandideh et al. [35] to optimize a multiproduct multiperiod three-echelon supply chain problem under uncertainty. Govindan et al. [15] proposed a robust hybrid multiobjective metaheuristic comprised of Electromagnetism-Like Algorithm (EMA) and VNS as a solution approach to their SCND model.

Among the metaheuristics, EMA is a relatively new population-based algorithm proposed by Birbil and Fang [36] that has been applied to many optimization problems (such as set covering [37], machine scheduling [38], flowshop scheduling [39,40], project scheduling [41], periodic job-shop scheduling [42] SCND [15], and travelling salesman problem $[43,44])$ and has outperformed many other similar algorithms [37, 38, 41, 42, 45-47]. In addition, EMA can be easily augmented in order to further improve its performance $[15,40,48]$.

Simulated Annealing (SA) and Variable Neighborhood Search (VNS) are well-known, efficient, and widely used metaheuristic methods. SA was originally proposed by Kirkpatrick et al. [49] and was inspired by the annealing process of materials. SA has already been successfully applied to many problems; however, as a parameter-sensitive algorithm, it may fail to fully explore the search space; therefore, hybridization of SA is proposed to resolve this drawback [48, 50-55]. VNS, originally proposed by Mladenović and Hansen [56], utilizes more than a single neighborhood structure and switches between them in a local search process. VNS is a simple and effective algorithm; however, it may get trapped in local minima due to limited exploration; therefore, hybridization of VNS is proposed to overcome this weakness [15, 57-62].

In this paper, a hybrid of EMA and SA and a combination of EMA and VNS are studied for solving the MINLP model of SCND problem under uncertainty and traffic congestion. In these hybrid algorithms the simple local search procedure in EMA is replaced by SA and VNS. The results show that 
the replacement leads to promising improvements. The main contributions of this research are as follows:

(1) concurrent consideration of multiple products, multiple transportation vehicles, multiple periods, time value of money, traffic congestion, and demand-side and supply-side uncertainties along with the time varying interest rate (time value of money) in SCND,

(2) proposing and studying two hybrid population-based metaheuristics (EMA-VNS and EMA-SA) for solving MINLP model of the SCND problem.

In order to clarify the characteristics of our model, Table 1 provides a comparison between the present study and the recent related works. The abbreviations used in this table are defined below the table. From the literature review point of view, it can be concluded that the literature on supply-side uncertainty, traffic congestion, and multiple transportation modes in SCND is scares compared to other features. In addition, to the best of the authors knowledge, concurrent consideration of all the features mentioned in Table 1 has not been studied. Furthermore, in the majority of the previously published researches, exact algorithms (e.g., CPLEX or LINGO solvers) are utilized. These methods may fail to efficiently solve complex problems. Therefore, a lack of study on metaheuristic methods for SCND problem with the aforementioned feature may be concluded from Table 1 . The literature on general SCND problem is immense; therefore, in this table, an effort is made to consider the most applicable features of SCND and most related works from the research background.

The rest of this paper is organized as follows. The next section provides a brief discussion on the concept of superiority and inferiority for fuzzy triangular numbers. In Section 3, the problem is presented and Section 4 discusses the solution methods. The results of numerical experiments are provided in Section 5 and, finally, Section 6 concludes the paper and presents guidelines for future research.

\section{Inferiority and Superiority of Fuzzy Triangular Numbers}

Fuzzy numbers may be utilized for modeling the uncertainty when the data is imprecise. The ease of application and the wide range of real problems in which they can be expressed through fuzzy triangular numbers are a good reason for their popularity among both researchers and practitioners.

In 2007, van Hop proposed an approach for comparing the fuzzy triangular numbers [63]. According to this theorem, if $\widetilde{P} \leq \widetilde{Q}$, then the superiority of a fuzzy triangular number $\widetilde{P}=(u, a, b)$ over a fuzzy triangular number $\widetilde{Q}=(v, c, d)$ is defined by the following equation:

$$
S(\widetilde{Q}, \widetilde{P})=v-u+\frac{d-b}{2} .
$$

In the above equation, $u$ is the center and $a$ and $b$ are the left and right spreads of $\widetilde{P}=(u, a, b)$, respectively, and $v$ is the center and $c$ and $d$ are the left and right spreads of
$\widetilde{Q}=(v, c, d)$, respectively. Similarly, the inferiority of $\widetilde{P}$ to $\widetilde{Q}$ is calculated by the following equation:

$$
I(\widetilde{P}, \widetilde{Q})=v-u+\frac{a-c}{2} .
$$

The concepts of superiority and inferiority can be suitably utilized for modeling the constraints of an optimization problem where a fuzzy triangular number is involved [64].

In this paper, the products demand and supply capacities are considered as triangular fuzzy numbers and the superiority and inferiority concepts are utilized for modeling "the inferiority of the demand for a product in a demand node in a time period to the amount of that product transported to that node" and "the superiority of the capacity for a product in a supply node in a time period over the amount of that product transported from that node." These are discussed in more details in Sections 3.3 and 3.4.

\section{The Problem}

In this section, a definition of the problem is presented, the assumptions based on which the mathematical model is built are provided, and the mathematical model is studied.

3.1. Problem Definition. In this paper, our focus is on facility location and transportation planning in SCND. Specifically, we assume a two-echelon supply chain which utilizes multiple vehicle types to transport multiple products to its customers in multiple planning periods. Here, for the sake of simplicity, two echelons are considered; however, the number of echelons may be extended to create a more realistic and complex model with some minor modifications. The problem is the location and relocation of facilities and determining the amounts of products transported from each facility to each customer in each planning period such that the total cost is minimized. The total cost consists of the facility location and relocation cost, the transportation cost, the traffic congestion cost, and the uncertainty cost. Here, the traffic congestion cost is calculated through the formula provided by the US Bureau of Public Roads (BPR) (formulated in (16)) and is expressed through the monetary value of time and the uncertainty cost is determined through the concepts of inferiority/superiority of the amounts of products transported from the facilities to the demand nodes to/over the uncertain demand and supply amounts as triangular fuzzy numbers. The formulations of these concepts are provided later in this section.

3.2. Assumptions. Assumptions determine the scope of the problem and define its capabilities and limitations. Current research is based on the following assumptions.

(1) The total number of nodes in the supply network, the total number of transportation vehicle types, the total number of products, the total number of periods in the planning horizon are known and fixed.

(2) The products are produced and shipped in continuous quantities. 
TABLE 1: A comparison of the recent related literature and our research (chronologically sorted).

\begin{tabular}{|c|c|c|c|c|c|c|c|c|c|c|c|}
\hline \multirow{2}{*}{ Author(s) } & & \multirow{2}{*}{ MP } & \multicolumn{2}{|c|}{ Uncertainty } & \multirow{2}{*}{ DY } & \multirow{2}{*}{$\mathrm{CN}$} & \multirow{2}{*}{ MM } & \multicolumn{3}{|c|}{ Network flow } & \multirow[t]{2}{*}{ Solution approach } \\
\hline & & & DU & SU & & & & FF & $\mathrm{RF}$ & $\mathrm{CL}$ & \\
\hline Liu et al. [65] & 2010 & & $\checkmark$ & & & & & $\checkmark$ & & & LR \\
\hline Rentizelas and Tatsiopoulos [66] & 2010 & $\checkmark$ & & & $\checkmark$ & & & $\checkmark$ & & & $\mathrm{GA}+\mathrm{SQP}$ \\
\hline Salema et al. [67] & 2010 & $\checkmark$ & & & $\checkmark$ & & & $\checkmark$ & $\checkmark$ & & CPLEX \\
\hline Pishvaee and Torabi [23] & 2010 & & $\checkmark$ & & $\checkmark$ & & & & & $\checkmark$ & LINGO \\
\hline Le and Lee [68] & 2013 & $\checkmark$ & & & $\checkmark$ & & $\checkmark$ & $\checkmark$ & & & LINGO \\
\hline Bai et al. [69] & 2011 & $\checkmark$ & & & & $\checkmark$ & & $\checkmark$ & & & $\mathrm{LPR}+\mathrm{LRB}$ \\
\hline Mirzapour Al-e-hashem et al. [70] & 2011 & $\checkmark$ & & & $\checkmark$ & & & $\checkmark$ & & & LINGO \\
\hline Georgiadis et al. [19] & 2011 & $\checkmark$ & $\checkmark$ & & $\checkmark$ & & & $\checkmark$ & & & CPLEX \\
\hline Pishvaee et al. [71] & 2011 & & $\checkmark$ & & & & & & & $\checkmark$ & CPLEX \\
\hline Chen and Fan [72] & 2012 & $\checkmark$ & $\checkmark$ & $\checkmark$ & & & & $\checkmark$ & & & DA \\
\hline Wang et al. [73] & 2011 & $\checkmark$ & & & & & & $\checkmark$ & & & CPLEX \\
\hline Kostin et al. [74] & 2012 & $\checkmark$ & & & $\checkmark$ & & $\checkmark$ & $\checkmark$ & & & SAA \\
\hline Almansoori and Shah [75] & 2012 & & $\checkmark$ & & $\checkmark$ & & $\checkmark$ & $\checkmark$ & & & CPLEX \\
\hline Bashiri et al. [76] & 2012 & $\checkmark$ & & & $\checkmark$ & & & $\checkmark$ & & & CPLEX \\
\hline Wang and Watada [77] & 2012 & & $\checkmark$ & & & & & $\checkmark$ & & & PSO \\
\hline Sadjady and Davoudpour [78] & 2012 & $\checkmark$ & & & & & $\checkmark$ & $\checkmark$ & & & LR \\
\hline Vahdani et al. [79] & 2013 & $\checkmark$ & & & & & & & & $\checkmark$ & GAMS \\
\hline Baghalian et al. [80] & 2013 & $\checkmark$ & $\checkmark$ & & & & & $\checkmark$ & & & LINGO \\
\hline Cardoso et al. [21] & 2013 & & $\checkmark$ & & $\checkmark$ & & & $\checkmark$ & $\checkmark$ & & CPLEX \\
\hline Ramezani et al. [25] & 2013 & & $\checkmark$ & $\checkmark$ & $\checkmark$ & & & $\checkmark$ & & & CPLEX \\
\hline Hatefi and Jolai [81] & 2014 & & $\checkmark$ & & & & & $\checkmark$ & $\checkmark$ & & CPLEX \\
\hline Jindal and Sangwan [82] & 2013 & $\checkmark$ & $\checkmark$ & $\checkmark$ & & & & & & $\checkmark$ & LINGO \\
\hline Jouzdani et al. [7] & 2013 & & $\checkmark$ & & $\checkmark$ & $\checkmark$ & & $\checkmark$ & & & LINGO \\
\hline Ramezani et al. [25] & 2013 & $\checkmark$ & $\checkmark$ & & & & & & & $\checkmark$ & SRA \\
\hline Soleimani et al. [83] & 2013 & $\checkmark$ & & & $\checkmark$ & & & & & $\checkmark$ & GA \\
\hline Yang and Liu [22] & 2015 & $\checkmark$ & $\checkmark$ & & & & & $\checkmark$ & & & MA \\
\hline Hasani et al. [11] & 2014 & $\checkmark$ & $\checkmark$ & $\checkmark$ & $\checkmark$ & & & & & $\checkmark$ & $\mathrm{MA}+\mathrm{VNS}$ \\
\hline Jabbarzadeh et al. [84] & 2014 & & $\checkmark$ & $\checkmark$ & $\checkmark$ & & & $\checkmark$ & & & LINGO \\
\hline Mousavi et al. [32] & 2014 & $\checkmark$ & & & $\checkmark$ & & & $\checkmark$ & & & Modified PSO \\
\hline Osmani and Zhang [10] & 2014 & & $\checkmark$ & $\checkmark$ & $\checkmark$ & & & $\checkmark$ & & & SAA \\
\hline Pishvaee et al. [5] & 2014 & & $\checkmark$ & $\checkmark$ & & & & & & $\checkmark$ & Benders Dec. \\
\hline Boukherroub et al. [13] & 2015 & $\checkmark$ & $\checkmark$ & & & & & & $\checkmark$ & & GA \\
\hline Sadjadi et al. [9] & 2014 & $\checkmark$ & $\checkmark$ & & & & & & $\checkmark$ & & MA \\
\hline Shabani et al. [85] & 2014 & $\checkmark$ & & $\checkmark$ & $\checkmark$ & & & $\checkmark$ & & & CPLEX \\
\hline Brandenburg [14] & 2015 & $\checkmark$ & & & $\checkmark$ & & & & & $\checkmark$ & $\mathrm{GA}+\mathrm{PSO}$ \\
\hline Dubey and Gunasekaran [12] & 2014 & $\checkmark$ & & & & & & & & $\checkmark$ & CPLEX \\
\hline Brandenburg [14] & 2015 & $\checkmark$ & $\checkmark$ & & $\checkmark$ & & $\checkmark$ & $\checkmark$ & & & CPLEX \\
\hline Boukherroub et al. [13] & 2015 & $\checkmark$ & & & $\checkmark$ & & $\checkmark$ & $\checkmark$ & & & CPLEX \\
\hline Govindan et al. [15] & 2015 & & $\checkmark$ & & & & & $\checkmark$ & & & $\mathrm{EMA}+\mathrm{VNS}$ \\
\hline Pasandideh et al. [35] & 2015 & $\checkmark$ & $\checkmark$ & $\checkmark$ & $\checkmark$ & & & $\checkmark$ & & & NSGA-II \\
\hline Current Research & & $\checkmark$ & $\checkmark$ & $\checkmark$ & $\checkmark$ & $\checkmark$ & $\checkmark$ & $\checkmark$ & & & Hybrid EMAs \\
\hline
\end{tabular}

MP: multiple products, DU: demand uncertainty, SU: supply uncertainly, DY: dynamic model (multiple periods), CN: congestion, MM: multiple transportation modes, FF: forward network flow, RF: reverse network flow, and CL: closed-loop network. 
(3) Each facility has a limited capacity for each product in each period. The capacity is subject to uncertainty and can be expressed through fuzzy triangular numbers.

(4) Each demand node has a limited demand for each product in each period. The demand is subject to uncertainty and can be expressed through fuzzy triangular numbers.

(5) All parameters except the capacity of vehicles and their traffic congestion coefficients are time varying.

3.3. Nomenclature. In order to facilitate the understanding of the mathematical model, the sets, parameters, and the decision variables are introduced in this section.

\subsubsection{Sets}

$N$ : the set of network nodes (including candidate facility locations and demand points),

$T$ : the set of time periods in the planning horizon,

$P$ : the set of products,

$M$ : the set of transportation modes.

\subsubsection{Subscripts}

$i$ : subscript for candidate facility location $(i \in N)$,

$j$ : subscript for demand point $(j \in N)$,

$t$ : subscript for time period $(t \in T)$,

$p$ : subscript for product $(p \in P)$,

$m$ : subscript for transportation vehicle $(m \in M)$.

\subsubsection{Parameters}

$\mathrm{IR}_{t}$ : interest rate in time period $t \in T$,

$\mathrm{MVT}_{t}:$ monetary value of time in time period $t \in T$,

$\alpha$ : parameter in the link performance function,

$\beta$ : parameter in the link performance function,

$\mathrm{CAP}_{m}$ : capacity of vehicle $m \in M$,

TCC $_{m}$ : traffic congestion coefficient of vehicle $m \in$ $M$,

$\mathrm{MC}_{i t}$ : the annual maintenance cost of a facility in candidate location $i \in N$ in time period $t \in T$,

$\mathrm{OC}_{i t}$ : the opening cost of a facility in candidate location $i \in N$ in time period $t \in T$,

$\mathrm{CC}_{i t}$ : the closing cost of a facility in candidate location $i \in N$ in time period $t \in T$,

$D_{j p t}^{c}$ : the center of the triangular fuzzy demand for product $p \in P$, in demand node $j \in N$, in time period $t \in T$,

$D_{j p t}^{\mathrm{ls}}$ : the left spread of the triangular fuzzy demand for product $p \in P$, in demand node $j \in N$, in time period $t \in T$,
$D_{j p t}^{\mathrm{rs}}:$ the right spread of the triangular fuzzy demand for product $p \in P$, in demand node $j \in N$, in time period $t \in T$,

$S_{i p t}^{c}$ : the center of the triangular fuzzy supply for product $p \in P$, in facility node $i \in N$, in time period $t \in T$,

$S_{i p t}^{\mathrm{ls}}:$ the left spread of the triangular fuzzy supply for product $p \in P$, in facility node $i \in N$, in time period $t \in T$,

$S_{i p t}^{\text {rs }}$ : the right spread of the triangular fuzzy supply for product $p \in P$, in facility node $i \in N$, in time period $t \in T$,

FFTT $_{i j t}$ : free flow travel time from node $i \in N$ to node $j \in N$ in time period $t \in T$,

$\mathrm{TCAP}_{i j t}$ : traffic capacity of the link from node $i \in N$ to node $j \in N$ in time period $t \in T$,

$\mathrm{BF}_{i j t}$ : basic traffic flow of the link from node $i \in N$ to node $j \in N$ in time period $t \in T$,

$C_{i j m t}$ : the transportation cost for vehicle $m \in M$ for link from node $i \in N$ to node $j \in N$ in time period $t \in T$,

$C_{I\left(D, x_{j p t}^{\mathrm{in}}\right)}$ : the unit cost of inferiority of the demand in a demand node $j \in N$ for product $p \in P$ in time period $t \in T$ to the amount of that product transported to that node,

$C_{S\left(S, x_{i p t} \text { out }\right)}$ : the unit cost of superiority of the capacity in a supply node $i \in N$ for product $p \in P$ in time period $t \in T$ over the amount of that product transported from that node.

\subsubsection{Decision Variables}

$y_{i t}$ : a binary variable which is equal to 1 if a facility is operating in $i \in N$ in time period $t \in T$ and equals 0 otherwise,

$x_{i j m p t}$ : the amount of product $p \in P$ transported from node $i \in N$ to node $j \in N$ by means of transportation vehicle $m \in M$ in time period $t \in T$.

\subsubsection{Auxiliary Variable}

$\lambda_{I\left(D, x_{j p t}^{\text {in }}\right)}$ : the inferiority of the demand in a demand node $j \in N$ for product $p \in P$ in time period $t \in T$ to the amount of that product transported to that node,

$\lambda_{S\left(S, x_{i p t}^{\text {out }}\right)}$ : the superiority of the capacity in a supply node $i \in N$ for product $p \in P$ in time period $t \in T$ over the amount of that product transported from that node,

$z_{i t}^{o}$ : being 1 if a facility is opened in candidate supply location $i \in N$ in time period $t \in T$, 
$z_{i t}^{c}$ : being 1 if a facility is closed in candidate supply location $i \in N$ in time period $t \in T$,

$n_{\text {ijmpt }}$ : the number of vehicles of type $m \in M$ transporting product $p \in P$ from node $i \in N$ to node $j \in N$ in time period $t \in T$ minus 1 ,

$z_{i j m t}$ : the total number of vehicles of type $m \in M$ transporting goods from node $i \in N$ to node $j \in N$ in time period $t \in T$,

$z_{i j m p t}^{l}:$ the auxiliary variable used form linearization, determining the amount of product $p \in P$ transported by vehicle of type $m \in M$ from node $i \in N$ to node $j \in N$ in time period $t \in T$ if a facility is operating in node $i \in N$,
$\mathrm{FLW}_{i j t}$ : total traffic flow from node $i \in N$ to node $j \in$ $N$,

$x_{j p t}^{\text {in }}$ : the total amount of product $p \in P$ transported to a node $j \in N$ in time period $t \in T$,

$x_{i p t}^{\text {out }}$ : the total amount of product $p \in P$ transported from a node $i \in N$ in time period $t \in T$.

3.4. Mathematical Model. In what follows, the dynamic multiproduct multimode SCND problem under transportation cost uncertainty is formulated according to the notations introduced above:

$$
\begin{aligned}
& \min \sum_{i \in N} \sum_{t \in T} y_{i t} \frac{\mathrm{MC}_{i t}}{\prod_{n=1}^{t}\left(1+\mathrm{IR}_{n}\right)}+\sum_{i \in N} \sum_{t \in T} z_{i t}^{o} \frac{\mathrm{OC}_{i t}}{\prod_{n=1}^{t}\left(1+\mathrm{IR}_{n}\right)}+\sum_{i \in N} \sum_{t \in T} z_{i t}^{c} \frac{\mathrm{CC}_{i t}}{\prod_{n=1}^{t}\left(1+\mathrm{IR}_{n}\right)} \\
& +\sum_{i \in N} \sum_{j \in N} \sum_{m \in M} \sum_{t \in T} \frac{C_{i j m t} z_{i j m t}}{\prod_{n=1}^{t}\left(1+\mathrm{IR}_{n}\right)} \\
& +\sum_{i \in N} \sum_{j \in N} \sum_{t \in T} \frac{\mathrm{MVT}_{t} \times \mathrm{FLW}_{i j t} \times t\left(\mathrm{FLW}_{i j t}\right)}{\prod_{n=1}^{t}\left(1+\mathrm{IR}_{n}\right)} \\
& +\sum_{j \in N} \sum_{p \in P} \sum_{t \in T} \frac{C_{I\left(D, x_{j p t}^{\text {in }}\right)} \times \lambda_{I\left(D, x_{j p t}^{\text {in }}\right)}}{\prod_{n=1}^{t}\left(1+\mathrm{IR}_{n}\right)} \\
& +\sum_{i \in N} \sum_{p \in P} \sum_{t \in T} \frac{C_{S\left(S, x_{i p t}^{\text {out }}\right)} \times \lambda_{S\left(S, x_{i p t}^{\text {out }}\right)}}{\prod_{n=1}^{t}\left(1+\mathrm{IR}_{n}\right)} \\
& z_{i t}^{c}=y_{i t} \quad \forall i \in N, t=|T|
\end{aligned}
$$




$$
\begin{aligned}
& y_{i t}, z_{i t}^{c}, z_{i t}^{o} \in\{0,1\} \quad \forall i \in N, \forall t \in T \\
& x_{i j m p t} \geq 0 \quad \forall i, j \in N, \forall p \in P, \forall m \in M, \forall t \in T .
\end{aligned}
$$

In the above model, (3a) calculates the total facility cost which consists of annual maintenance cost, opening cost, and closing cost of facilities in the SC. Equation (3b) presents the total transportation cost. Total traffic congestion cost is calculated in (3c) in which the following formula is provided by the US Bureau of Public Roads (BPR) as

$$
\begin{aligned}
& t\left(\mathrm{FLW}_{i j t}\right)=\operatorname{FFTT}_{i j t}\left(1+\alpha\left(\frac{\mathrm{FLW}_{i j t}}{\mathrm{TCAP}_{i j t}}\right)^{\beta}\right), \\
& \forall i, j \in N, \forall t \in T .
\end{aligned}
$$

Equation (3d) calculates the cost of inferiority of the demand in demand nodes to the amounts of products transported to those nodes. Similarly, (3e) is for obtaining the cost of superiority of the capacity in supply nodes over the amounts of products transported from those nodes.

Constraint (4) and Constraint (5) determine the total number of each vehicle type used for transporting goods from each node to another in each time period. Constraint (6) is for obtaining the flow of each link on each time period based on its basic flow and the congestion caused by the fleet of the SC. Constraint (7) presents the flow balance in each node. Constraint (8) calculates the inferiority of the demand in each demand node for each product in each time period to the amount of that product transported to that node. Similarly, Constraint (9) gives the superiority of the capacity in each supply node for each product in each time period over the amount of that product transported from that node. In Constraint (10), binary variables are defined to determine if a facility is opened in each node in each time period and Constraint (11) initializes the variables for the first time period in the planning horizon. Similarly, Constraint (12) calculates binary variables that determine if a facility is closed in each node in each time period and Constraint (13) determines the values for the final time period in the planning horizon. Finally, Constraint (14) and Constraint (15) determine the types of variables.

\section{The Solution Algorithms}

The proposed algorithms are hybridization of the Electromagnetism-Like Algorithm (EMA) with Simulated Annealing (SA) algorithm and the Variable Neighborhood Search (VNS), respectively. The hybrid algorithms and their aforementioned elements (i.e., EMA, SA, and VNS) are discussed in what follows.

4.1. The Electromagnetism-Like Algorithm. The EMA was originally proposed by Birbil and Fang [36]. The main idea in EMA was originated from the behavior of electromagnetic particles and the forces they exert on each other based on their distance and charge. The analogy here is that the solutions in the search space are considered as particles and their fitness values are regarded as their charges.

In Algorithm 1 popSize is the number of solution points (particles), maxIter is the maximum number of the algorithm iterations, LSIter is the maximum number of local search steps, and $i$ and $j$ are local counter variables.

The original scheme of EMA is presented in Algorithm 1 in which the forces are calculated according to Coulomb law [86] as

$$
F_{i j}=\frac{q_{i} q_{j}}{r_{i j}^{2}}, \quad \forall i, j \in\{1,2, \ldots, m\},
$$

where $F_{i j}$ is the force particle $i$ and particle $j$ exert on each other, $r_{i j}$ is the distance between those particles, $m$ is the number of particles, and $q_{i}$ and $q_{j}$ are the electrical charges of particle $i$ and particle $j$, respectively. In EMA, the charges of the particles are calculated as

$$
\begin{aligned}
& q_{i}=e^{-n\left(\left(f\left(x_{i}\right)-f\left(x_{\text {best }}\right)\right) /\left(\sum_{k=1}^{m} f\left(x_{k}\right)-f\left(x_{\text {best }}\right)\right)\right)}, \\
& \forall i \in\{1,2, \ldots, m\},
\end{aligned}
$$

where $f(x)$ is the objective function value for solution $x$ and $n$ is the dimension of the solution space. The force exerted on each particle $i$ is then obtained according to the following equation:

$$
F_{i}= \begin{cases}\sum_{\substack{j=1 \\ j \neq i}}^{m}\left(x_{j}-x_{i}\right) F_{i j}, & \text { if } q_{i}<q_{j} \\ \sum_{\substack{j=1 \\ j \neq i}}^{m}\left(x_{i}-x_{j}\right) F_{i j}, & \text { if } q_{i} \geq q_{j},\end{cases}
$$

$\forall i \in\{1,2, \ldots, m\}$

The forces are used to move the particles in the solution space such that the updated particle is in the feasible solution space. The particles are moved according to the total force exerted on them through the following equation in which $\lambda$ randomizes the particle movement and $\left\|F_{i}\right\|$ is the norm of the force vector:

$$
x_{i} \longleftarrow x_{i}+\lambda \frac{F_{i}}{\left\|F_{i}\right\|}, \quad \forall i \in\{1,2, \ldots, m\} .
$$

Originally, EMA is designed for solving continuous optimization problems with bounded variables; however, many discrete problems such as machine scheduling [38, 87], cell formation [45], project scheduling [41], flow-shop scheduling [88], job-shop scheduling [42], assembly sequences planning [28], set covering [37], and travelling salesman problem [43, $44]$ are solved using the EMA. 


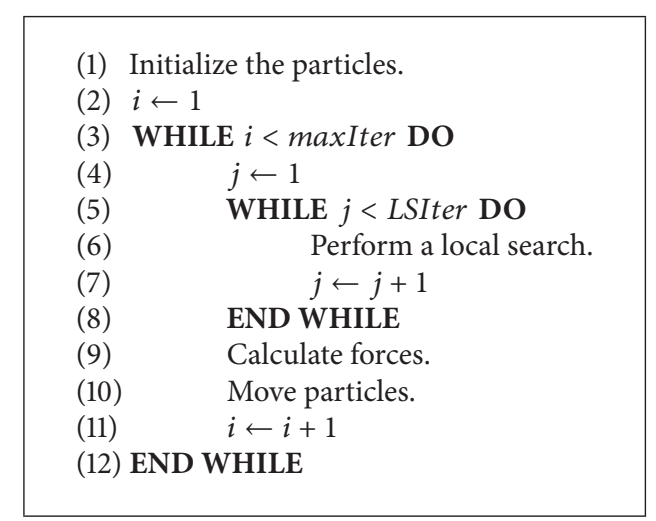

Algorithm 1: General procedure of the original EMA.

4.2. The Simulated Annealing Algorithm. Simulated Annealing was introduced by Kirkpatrick et al. [49]. The method is inspired by metal annealing process in which the material is heated in order to prepare the material for atomic restructure and then gradually cooled down to reach the desired atomic configuration. Here, the analogy is between the temperature in the real annealing process and the temperature in SA. The algorithm is capable of escaping the local optima by allowing the acceptance of worse solutions in each iteration based on its temperature. More specifically, the higher the temperature, the more the chance of the algorithm moving to a worse solution in hope of finding the optimum point in the search space. SA is capable of efficiently and effectively solving combinatorial optimization problem and is easy to implement [89]. However, as a parameter-sensitive trajectory method, SA may fail to fully explore the search space. Therefore, it is combined with other metaheuristics to compensate its lack of exploration [48, 53-55].

4.3. The Variable Neighborhood Search Algorithm. Trajectory methods deal with a single solution in each step; however, they have shown their capability in exploiting the promising subsets of search space in order to reach high quality solutions. VNS is a simple and yet effective trajectory search algorithm originally proposed by Mladenović and Hansen [56]. VNS utilizes more than a single neighborhood structure and switches between the structures in a local search process. Contrary to many other metaheuristics, VNS needs few or sometimes no parameters. Despite its capabilities, VNS may be trapped in local minima due to limited exploration. Hence, its hybridization with other metaheuristics is proposed in the literature and is recently applied to solve problems [15, 57-61].

4.4. Solution Representation. One of the major issues in any search algorithm is the solution representation. In order to encode solutions into a particle, a 5-dimensional matrix, $X$, of size $|N| \times|N| \times|M| \times|P| \times|T|$, where $|A|$ represents the number of elements in the set $A$, is considered. In $X$, each element $X[i, j, m, p, t]$ has the same interpretation as $x_{i j m p t}$. It should be noted that $X$ also contains the information about the decision variable $y_{i t}$. For example, consider the binary decision variable, $y_{23}$, which is equal to 1 if a facility is operating in node $2 \in N$ in time period $3 \in T$ and equals 0 otherwise. If $y_{23}=0$, then no facility is opened in node $2 \in N$ in time period $3 \in T$. Therefore, we have $x_{2 j m p 3}=0, \forall j \in N$, $\forall m \in M, \forall p \in P$, and apparently according to the definition of $X$, we set $X[2, j, m, p, 3]=0, \forall j \in N, \forall m \in M, \forall p \in P$.

4.5. The EMA-SA Hybrid. EMA results in satisfying solutions when combined with SA and has been successfully applied to discrete problems [40] and continuous search spaces [48]. In this section, an algorithm which benefits from both the EMA and SA is discussed. More specifically, the local search procedure in the original EMA is replaced by the SA. In addition, in order to provide the algorithm with a more guided search, the concept of temperature is incorporated into the movement of particles such that, in the early iterations of the algorithm, the particles move faster in order to increase the exploration of the search space, while in the final iterations, the temperature is dropped and the particles move slower in order to provide the intensification of the search procedure.

A neighborhood to a solution, $S$, in SA is a solution $S^{\prime}=$ $N_{1}(S)$ that is generated by adding a matrix $D$, with randomly generated element, of the same size as $S$ to $S$. By applying this type of neighborhood structure, some of the generated solutions may be infeasible. In such cases, the solutions are simply discarded. The general scheme of the EMA-SA hybrid for a minimization problem can be expressed as shown in Algorithm 2.

In Algorithm 2, the force particle $i$ and particle $j$ exert on each other, $F_{i j}$, is calculated as

$$
F_{i j}=k \frac{q_{i} q_{j}}{r_{i j}^{2}}, \quad \forall i, j \in\{1,2, \ldots, m\}
$$

where $k$ is obtained through the following equation and other elements are the same as defined in (17):

$$
k=1+\frac{T-T_{f}}{T_{0}-T_{f}} .
$$

It should be noted that, in EMA, we have $k=1$. The reason for using the coefficient in (22) is to incorporate the concept of temperature into the movement of particles. More specifically, as the temperature drops the force that the particles exert on each other decreases resulting in a more intense search in comparison with the early iterations of the algorithm. In addition, in a minimization problem, the charge for a particle $i$ is calculated as

$$
q_{i}=n \frac{f\left(x_{\text {worst }}\right)-f\left(x_{i}\right)}{f\left(x_{\text {worst }}\right)-f\left(x_{\text {best }}\right)}, \quad \forall i \in\{1,2, \ldots, m\},
$$

where $x_{\text {worst }}$ is the particle with the worst (largest) objective function and other elements are the same as defined in (18). The above equation not only considers the closeness to the best solution but also takes into account the distance from the worst particle. The calculation of $F_{i}$ and the movement of each particle are the same as in EMA. 


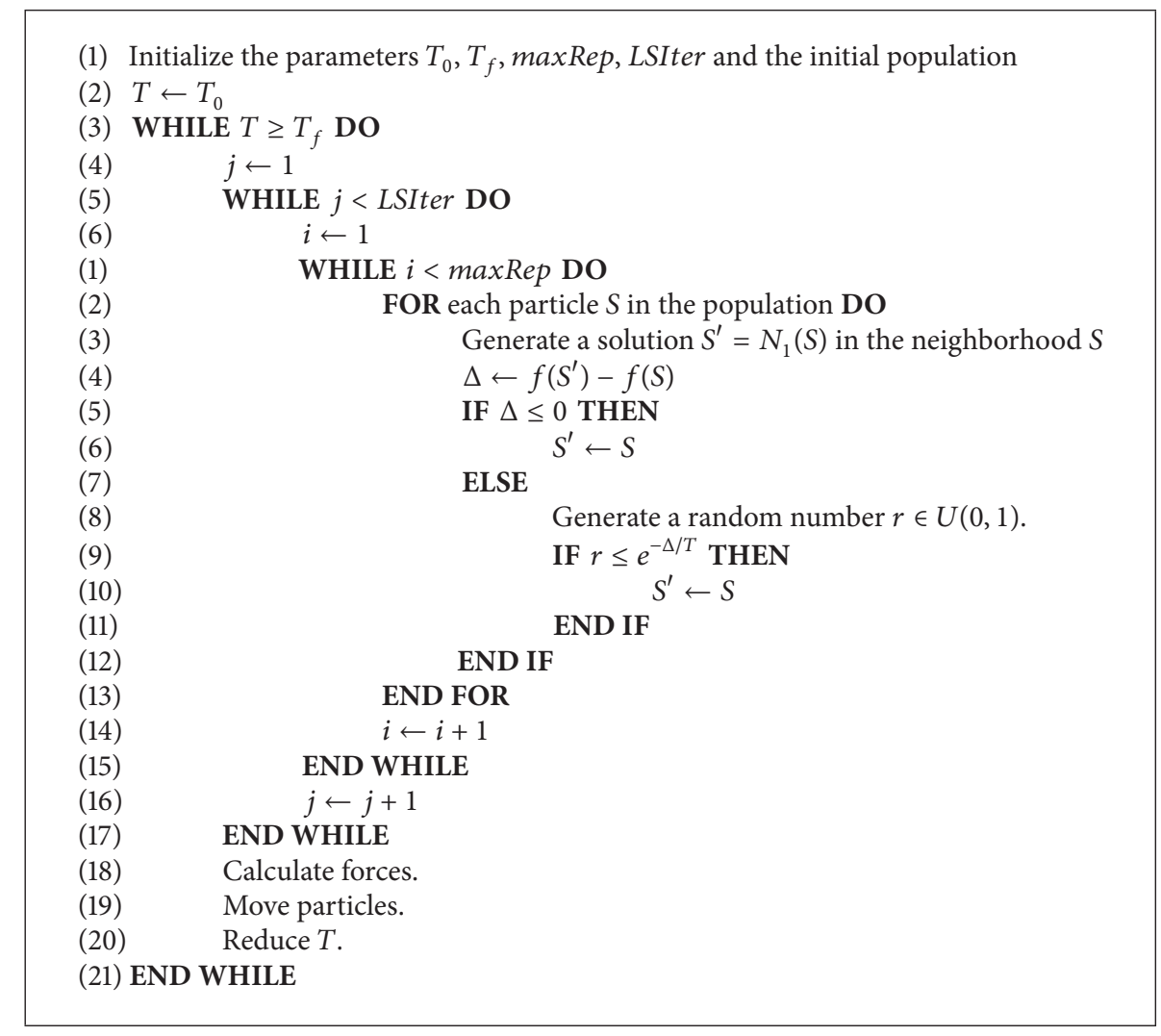

Algorithm 2: General procedure of the EMA-SA hybrid.

4.6. The EMA-VNS Hybrid. EMA and VNS have already been successfully combined for tackling problems [15]. Similar to what was mentioned in the previous section, we combine the EMA and VNS by replacing the simple local search in EMA by the VNS.

The neighborhood structure in VNS is simple: a neighbor to a solution $S$ is $S^{\prime}=N_{2}(S)$, which is generated by adding a matrix $D$ of the same size as $S$ to $S$. The elements of $D$ are normally distributed random variables with a mean of their corresponding elements in $S$ and a variance of $\sigma$ which is an increasing function of, $i$, the VNS iteration number, denoted by $v(i)$. Similar to what was mentioned above, the infeasible generated neighbors are discarded. Therefore, the general scheme of the EMA-VNS hybrid for a minimization problem can be expressed in Algorithm 3 in which other calculations are as in what was mentioned regarding the original EMA.

\section{Numerical Results}

5.1. A Small Test Problem. In order to investigate the proposed model, a small test problem of size $4 \times 4 \times 2 \times 2 \times 2$, that is, 4 network nodes, 2 transportation modes, 2 products, and 2 periods, is studied. The problem is coded into LINGO 9.0 and solved by using a PC equipped with an Intel Atom CPU N455@1.66 GHz and 2.00 GB of RAM running Windows 7 Starter operating system. The obtained cost components are presented in Table 2 and the optimal solution is depicted in Tables 3 and 4 and illustrated in Figure 1. It should be noted that the demand for both products in period 1 in City 3 is
TABLE 2: The obtained results for the global optimal solution to the small test problem.

\begin{tabular}{lc}
\hline Cost component & Value \\
\hline Total system cost & 184.15 \\
Total facility maintenance cost & 3.39 \\
Total facility opening cost & 101.02 \\
Total facility closing cost & 6.01 \\
Total demand violation cost & - \\
Total supply violation cost & 11.79 \\
Total transportation cost & 37.22 \\
Total congestion cost & 24.73 \\
\hline
\end{tabular}

TABLE 3: Facility open/close status for planning periods.

\begin{tabular}{lcc}
\hline Facilities & Period 1 & Period 2 \\
\hline City 1 & $\boldsymbol{x}$ & $\boldsymbol{x}$ \\
City 2 & $\checkmark$ & $\boldsymbol{x}$ \\
City 3 & $\checkmark$ & $\checkmark$ \\
City 4 & $\boldsymbol{x}$ & $\boldsymbol{x}$ \\
\hline
\end{tabular}

satisfied by the facility opened in that node. It is interesting to see that the configuration of the network is changed in the second time period in a way that the demand in City 1 is partially satisfied by products initially transported from City 3 to City 4 and then to City 1 . The obtained solution may be justified by the model parameter data presented in 


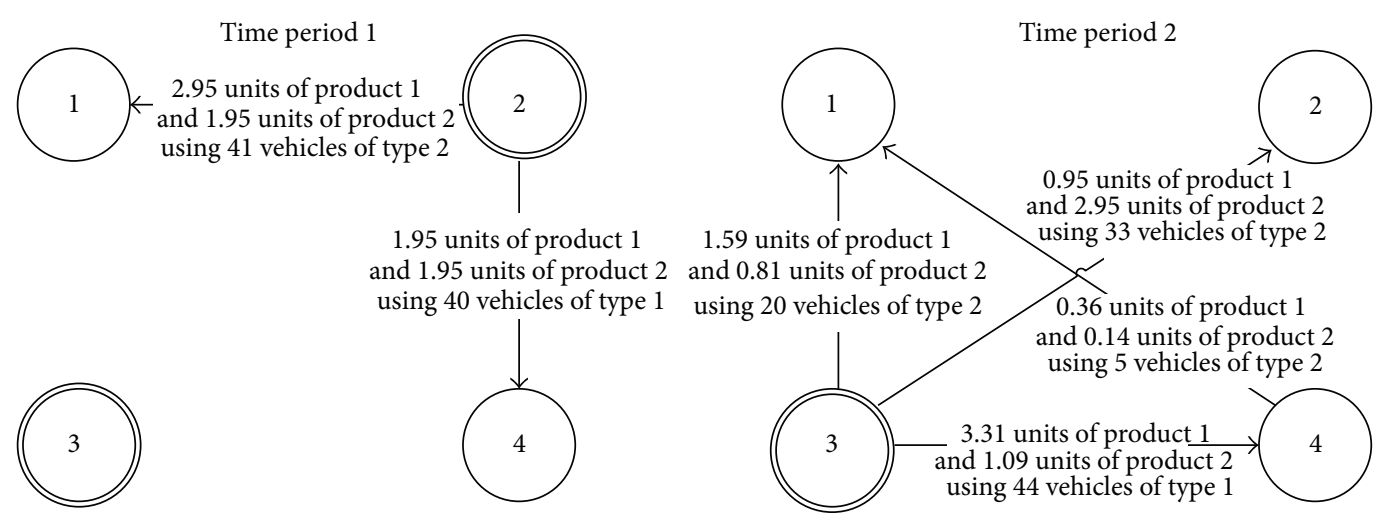

FIGURE 1: The illustration of the solution to the small problem.

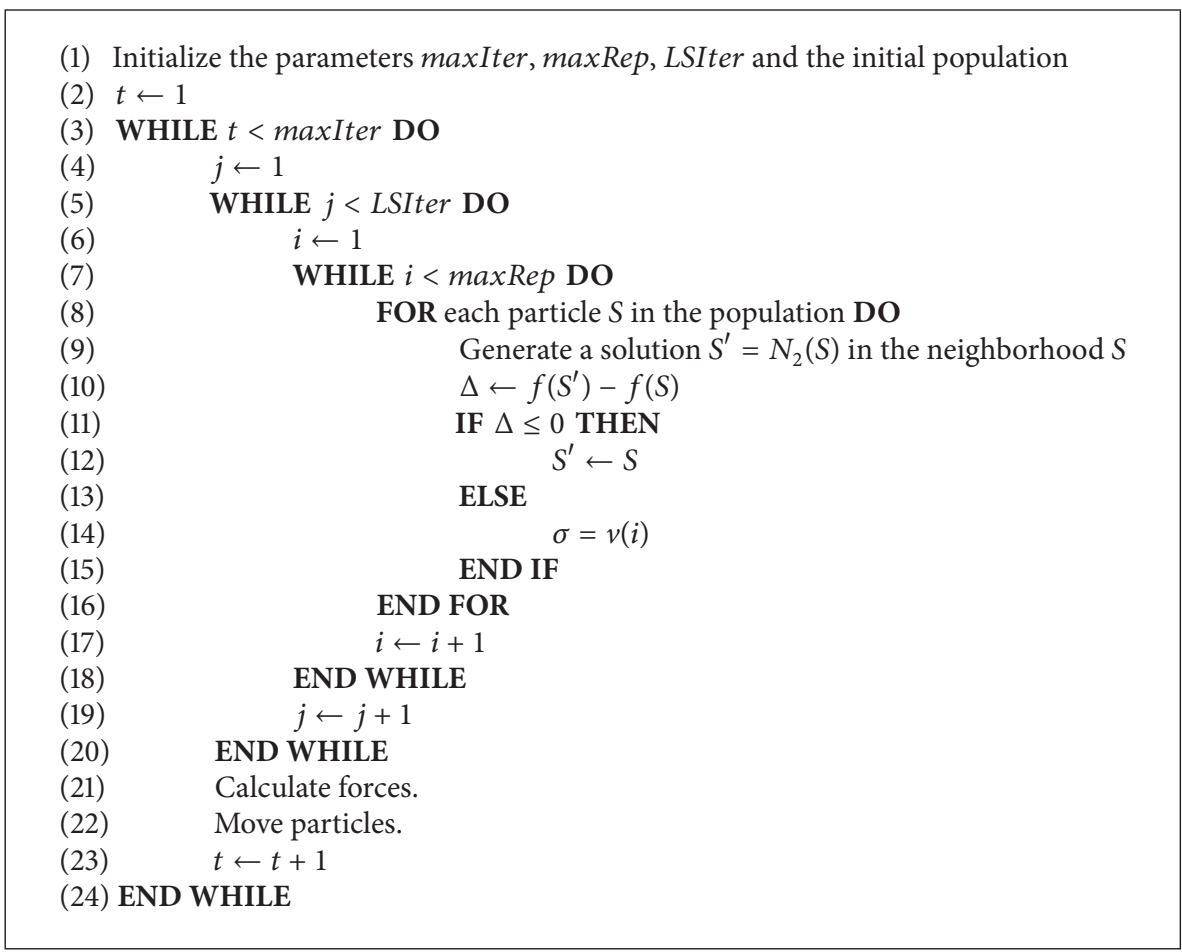

Algorithm 3: General procedure of the EMA-VNS hybrid.

the Appendix. The LINGO code is available upon requesting the corresponding author.

In order to shed light on the impact of congestion in SCND, the model is solved not considering the traffic congestion cost. The results, presented in Table 5, indicate that neglecting the congestion costs leads to an increase in the total system cost. Moreover, by not considering the congestion cost, total transportation cost decreases while the total congestion cost increases. This translates to the significant fact that slightly cheaper but more congested routes are selected for transportation of products, and/or cheaper transportation modes that have higher congestion costs are preferred.

Here, the monetary value of time plays a significant role. To show its effect, in another experiment the monetary value of time is increased from 0.01 to 0.05 for the same test problem. In this case, the result of neglecting the congestion cost is a $3.66 \%$ decrease in total transportation cost and $13.87 \%$ and $34.00 \%$ increase in the total system cost and total congestion cost, respectively. It is notable that the decrease in total transportation cost is smaller and the increase in the total system cost and the congestion cost is greater in comparison to what is presented in Table 5 where monetary value of time is 0.01 . Therefore, the higher the monetary value of time is, the more significant it is to consider the congestion cost in SCND.

5.2. Comprehensive Experiments. In order to justify the proposed model and the performance of the solution algorithm, several test problems are solved and the results are presented in this section. The test problems are categorized into three 
TABLE 4: Product flows for optimal solution.

\begin{tabular}{|c|c|c|c|c|c|c|c|c|c|}
\hline \multicolumn{2}{|c|}{ Product flows } & \multicolumn{4}{|c|}{ Period 1} & \multicolumn{4}{|c|}{ Period 2} \\
\hline \multirow[b]{2}{*}{ From } & \multirow[b]{2}{*}{ To } & \multicolumn{2}{|c|}{ Mode 1} & \multicolumn{2}{|c|}{ Mode 2} & \multicolumn{2}{|c|}{ Mode 1} & \multicolumn{2}{|c|}{ Mode 2} \\
\hline & & Product 1 & Product 2 & Product 1 & Product 2 & Product 1 & Product 2 & Product 1 & Product 2 \\
\hline \multirow{4}{*}{ City 1} & City 1 & - & - & - & - & - & - & - & - \\
\hline & City 2 & - & - & - & - & - & - & - & - \\
\hline & City 3 & - & - & - & - & - & - & - & - \\
\hline & City 4 & - & - & - & - & - & - & - & - \\
\hline \multirow{4}{*}{ City 2} & City 1 & - & - & 2.95 & 1.95 & - & - & - & - \\
\hline & City 2 & - & - & - & - & - & - & - & - \\
\hline & City 3 & - & - & - & - & - & - & - & - \\
\hline & City 4 & 1.95 & 1.95 & - & - & - & - & - & - \\
\hline \multirow{4}{*}{ City 3} & City 1 & - & - & - & - & - & - & 1.59 & 0.81 \\
\hline & City 2 & - & - & - & - & - & - & 0.95 & 2.95 \\
\hline & City 3 & - & - & - & - & - & - & - & - \\
\hline & City 4 & - & - & - & - & 3.31 & 1.09 & - & - \\
\hline \multirow{4}{*}{ City 4} & City 1 & - & - & - & - & - & - & 0.36 & 0.14 \\
\hline & City 2 & - & - & - & - & - & - & - & - \\
\hline & City 3 & - & - & - & - & - & - & - & - \\
\hline & City 4 & - & - & - & - & - & - & - & - \\
\hline
\end{tabular}

TABLE 5: The impact of traffic congestion in SCND problem.

\begin{tabular}{lccc}
\hline Cost component & Value & Value (neglecting congestion) & Relative percent change \\
\hline Total system cost & 184.15 & 189.21 & $2.75 \%$ \\
Total facility maintenance cost & 3.39 & 3.39 & $0.00 \%$ \\
Total facility opening cost & 101.02 & 101.02 & $0.00 \%$ \\
Total facility closing cost & 6.01 & 6.01 & $0.00 \%$ \\
Total demand violation cost & - & - & $0.00 \%$ \\
Total supply violation cost & 11.79 & 11.79 & $0.00 \%$ \\
Total transportation cost & 37.22 & 35.78 & $-4.02 \%$ \\
Total congestion cost & 24.73 & 31.22 & $20.79 \%$ \\
\hline
\end{tabular}

TABle 6: The size characteristics of small, medium, and large test problems.

\begin{tabular}{lcccccccc}
\hline \multirow{2}{*}{ Category } & \multicolumn{2}{c}{$|N|$} & \multicolumn{2}{c}{$|M|$} & \multicolumn{2}{c}{$|P|$} & \multicolumn{2}{c}{$|T|$} \\
& Min & Max & Min & Max & Min & Max & Min & Max \\
\hline Small & 10 & 14 & 3 & 5 & 5 & 9 & 3 & 4 \\
Medium & 15 & 20 & 6 & 9 & 10 & 15 & 5 & 6 \\
Large & 11 & 25 & 10 & 13 & 16 & 20 & 7 & 10 \\
\hline
\end{tabular}

groups based on their sizes: small, medium, and large. The sizes of these test problems are shown in Table 6.

In order to further study different hybrids of EMA, a third algorithm comprised of EMA and Tabu Search (TS) is added to the comparison. Following the same structure explained for EMA-SA and EMA-VNS, the local search procedure in EMA is replaced with TS in EMA-TS hybrid. In each problem category, 50 test problems are randomly generated and solved 30 times by EMA-SA, EMA-VNS, and EMA-TS hybrids and the original EMA. The hybrid of EMA and VNS has been shown to outperform other well-known algorithms in the context of SCND [15]. Therefore, to demonstrate the performance of the proposed algorithms, they are used in the comparison. All algorithms are coded by MATLAB R2013a. In each problem category, the parameters of the algorithms are tuned for the largest problem in the category by using a factorial Design of Experiments (DOE) and the Response Optimizer in MINITAB 16.

The overall performances of algorithms are compared in Table 7 which provides the results for the experiments for small, medium, and large problems and the corresponding mean objective function values, the mean CPU times, and the gap from the best results in each criterion. The statistical significance of the gaps is tested by means of Wilcoxon and the results indicate that the minimum significance is $5 \%$ for the gaps. In addition, in this table, the best value in each criterion for each problem group is indicated by boldface numbers.

Generally, considering the solution quality, EMA-VNS outperforms other algorithms (at the cost of a larger computational time), while with less computational effort (at the cost of a lower solution quality), EMA performs better than other algorithms.

In order to demonstrate the evolution of the objective function, a problem instance from each problem category is 
TABLE 7: The comparison of the algorithms regarding the objective function and computational time.

\begin{tabular}{cccccc}
\hline Problem & Algorithm & OBJ & TIME & OBJ GAP & CPU GAP \\
\hline \multirow{4}{*}{ Small } & EMA-SA & $\mathbf{3 0 2 0 3 . 0}$ & 83.15 & $0.000 \%$ & $18.055 \%$ \\
& EMA & 39772.9 & $\mathbf{6 8 . 1 3}$ & $24.061 \%$ & $0.000 \%$ \\
& EMA-VNS & 30276.3 & 108.82 & $0.242 \%$ & $37.388 \%$ \\
& EMA-TS & 36967.8 & 75.91 & $18.299 \%$ & $10.238 \%$ \\
\hline \multirow{4}{*}{ Medium } & EMA-SA & 112212.6 & 1499.71 & $0.242 \%$ & $12.167 \%$ \\
& EMA & 123348.4 & $\mathbf{1 3 1 7 . 2 4}$ & $9.248 \%$ & $0.000 \%$ \\
& EMA-VNS & $\mathbf{1 1 1 9 4 1 . 0}$ & 1541.92 & $0.000 \%$ & $14.571 \%$ \\
& EMA-TS & 121293.9 & 1493.37 & $7.711 \%$ & $11.794 \%$ \\
\hline \multirow{4}{*}{ Large } & EMA-SA & 237645.1 & 9048.42 & $0.004 \%$ & $15.623 \%$ \\
& EMA & 250421.9 & $\mathbf{7 6 3 4 . 8 0}$ & $5.106 \%$ & $0.000 \%$ \\
& EMA-VNS & $\mathbf{2 3 7 6 3 6 . 1}$ & 9825.95 & $0.000 \%$ & $22.300 \%$ \\
& EMA-TS & 247392.1 & 10963.4 & $3.944 \%$ & $30.362 \%$ \\
\hline \multirow{4}{*}{ All } & EMA-SA & 124831.5 & 3477.21 & $0.053 \%$ & $15.156 \%$ \\
& EMA & 135961.7 & $\mathbf{2 9 5 0 . 2 1}$ & $8.235 \%$ & $0.000 \%$ \\
& EMA-VNS & $\mathbf{1 2 4 7 6 5 . 1}$ & 3754.09 & $0.000 \%$ & $21.413 \%$ \\
& EMA-TS & 133328.5 & 4090.02 & $6.423 \%$ & $27.868 \%$ \\
\hline
\end{tabular}

TABLE 8: The significance for Mann-Whitney test for MIC of the algorithms.

\begin{tabular}{lcccc}
\hline Problem & Algorithm & EMA-SA & EMA-VNS & EMA-TS \\
\hline \multirow{4}{*}{ Small } & EMA-SA & & & \\
& EMA-VNS & 0.0625 & & \\
& EMA-TS & 0.0050 & 0.0129 & \\
& EMA & 0.0005 & 0.0085 & 0.4001 \\
\hline \multirow{4}{*}{ Medium } & EMA-SA & & & \\
& EMA-VNS & 0.4725 & & \\
& EMA-TS & 0.0087 & 0.0105 & \\
Large & EMA & 0.0001 & 0.0001 & 0.1352 \\
\hline \multirow{4}{*}{ EMA-VNS } & 0.4047 & & \\
& EMA-TS & 0.0000 & 0.0000 & \\
Ell & EMA & 0.0002 & 0.0002 & 0.1086 \\
\hline \multirow{4}{*}{ EMA-SA } & & & \\
& EMA-VNS & 0.2568 & & \\
& EMA-TS & 0.0000 & 0.0000 & \\
& EMA & 0.0000 & 0.0000 & 0.1947 \\
\hline
\end{tabular}

TABLE 9: Transportation modes data.

\begin{tabular}{lcc}
\hline Modes & Mode traffic costs & Mode capacities \\
\hline Mode 1 & 1 & 0.1 \\
Mode 2 & 1.5 & 0.12 \\
\hline
\end{tabular}

solved by using all four algorithms. The algorithms are started with the same initial population of solutions. The evolution of the objective function value of the small, medium, and large problem instance in each algorithm is depicted in Figures 4, 3 , and 2, respectively. These figures show that the replacement of the local search procedure in the EMA with SA and VNS results in promising improvements in the quality of the obtained solutions.

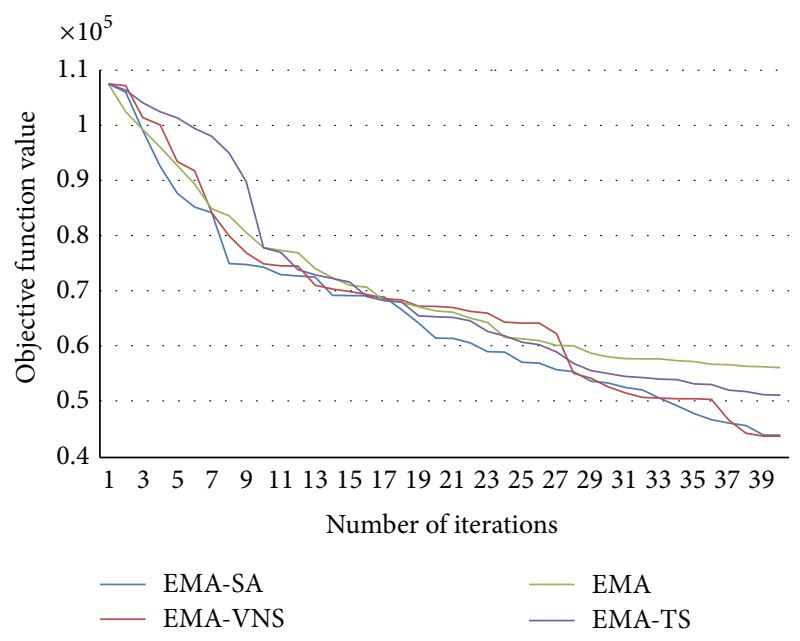

FIgURE 2: The evolution of the objective function of the small problem instance for the algorithms.

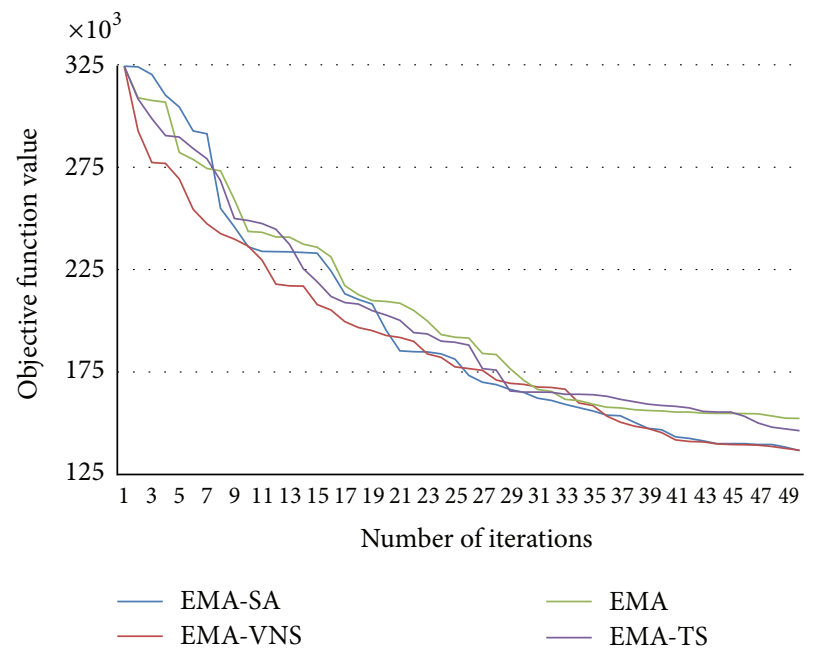

FIgURE 3: The evolution of the objective function of the medium problem instance for the algorithms.

In order to further investigate the performance of the four algorithms, a slightly modified version of the Marginal Improvement per CPU (MIC) criterion, originally proposed by Osman [90], is utilized. The MIC for an algorithm $\alpha$ in a problem instance $\rho$ is calculated by the following equation:

$$
\mathrm{MIC}_{\alpha \rho}=\frac{\mathrm{RPI}_{\alpha \rho}}{\mathrm{CPU}_{\alpha \rho}}, \quad \forall \alpha \in A, \forall \rho \in P
$$

In the above equation, $\mathrm{RPI}_{\alpha \rho}$ and $\mathrm{CPU}_{\alpha \rho}$ are the Relative Percent Improvement (RPI) and the CPU for algorithm $\alpha$ in a problem instance $\rho$, respectively. $A$ and $P$ are the set of algorithms and the set of problems, respectively. The RPI is given by

$$
\mathrm{RPI}_{\alpha \rho}=\frac{c_{W}^{\rho}-c_{B}^{\alpha \rho}}{c_{W}^{\rho}-c_{B}^{\rho}} \times 100, \quad \forall \alpha \in A, \quad \forall \rho \in P
$$


TABLE 10: The data for links traffic capacities.

\begin{tabular}{lcccccccc}
\hline \multirow{2}{*}{ Link traffic capacity } & \multicolumn{2}{c}{ City 1 } & \multicolumn{2}{c}{ City 2 } & \multicolumn{2}{c}{ City 3 } & \multicolumn{2}{c}{ City 4 } \\
& Period 1 & Period 2 & Period 1 & Period 2 & Period 1 & Period 2 & Period 1 & Period 2 \\
\hline City 1 & 76 & 56 & 68 & 54 & 36 & 20 & 72 \\
City 2 & 44 & 72 & 58 & 44 & 60 & 40 & 42 \\
City 3 & 64 & 32 & 34 & 46 & 62 & 48 & 36 \\
City 4 & 70 & 50 & 56 & 30 & 32 & 32 & 48 \\
\hline
\end{tabular}

TABLE 11: The data for links basic flows.

\begin{tabular}{lcccccccc}
\hline \multirow{2}{*}{ Link basic flow } & \multicolumn{2}{c}{ City 1 } & \multicolumn{2}{c}{ City 2 } & \multicolumn{2}{c}{ City 3 } & \multicolumn{2}{c}{ City 4 } \\
& Period 1 & Period 2 & Period 1 & Period 2 & Period 1 & Period 2 & Period 1 & Period 2 \\
\hline City 1 & 6 & 7 & 2 & 5 & 4 & 1 & 4 \\
City 2 & 2 & 2 & 10 & 6 & 1 & 6 & 5 \\
City 3 & 3 & 8 & 5 & 1 & 7 & 3 & 6 \\
City 4 & 9 & 8 & 1 & 3 & 8 & 7 & 4 \\
\hline
\end{tabular}

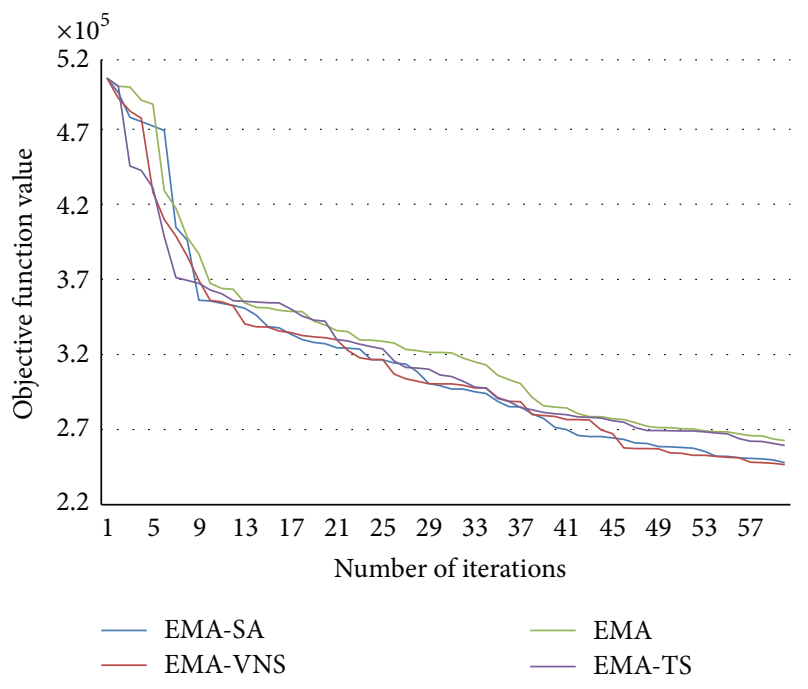

FIGURE 4: The evolution of the objective function of the large problem instance for the algorithms.

in which $c_{B}^{\rho}$ and $c_{W}^{\rho}$ are the best and the worst known objective function values for problem instance $\rho$, respectively, and $c_{B}^{\alpha \rho}$ is the best objective function value for problem $\rho$ obtained by using algorithm $\alpha$.

In order to compare the algorithm by using the MIC criterion, a Mann-Whitney test (two-sample Wilcoxon test) is utilized. The Mann-Whitney test is a nonparametric hypothesis test which is utilized to determine whether two populations have the same population median. This test does not require the data to be sampled from normally distributed populations. The $P$ values for Mann-Whitney test for the medians of MICs for each pair of algorithms in each problem category are given in Table 8 . In this table, each value represents the $P$ value for the following test:

$$
\begin{aligned}
& H_{0}: \eta_{c}=\eta_{r} \\
& H_{1}: \eta_{c}>\eta_{r},
\end{aligned}
$$

where $\eta_{c}$ is the median of the MIC of the algorithm in the column and $\eta_{r}$ is that of the one mentioned in the row of the table. For example, the significance for the test

$$
\begin{aligned}
& H_{0}: \eta_{\mathrm{EMA}-\mathrm{SA}}=\eta_{\mathrm{EMA}} \\
& H_{1}: \eta_{\mathrm{EMA}-\mathrm{SA}}>\eta_{\mathrm{EMA}}
\end{aligned}
$$

for the small problem category is equal to 0.0005 and can be found in the fifth row and third column of Table 8. From this $P$ value, it can be concluded that the null hypothesis of equality of the MIC median for EMA-SA with that of EMA is rejected with $99.95 \%$ confidence. Considering the alternative hypothesis in (27), it can be concluded that EMASA is superior to EMA according to MIC criterion.

From Table 8, it can be concluded that the difference between the MICs of EMA-SA and EMA-VNS is not statistically significant. However, according to this table, EMA-VNS and EMA-SA are both improvements of the EMA in terms of MIC criterion. Furthermore, the MICs of EMA-SA and EMA-VNS hybrids are higher than that of the hybrid of EMA and TS (EMA-TS) and the difference is statistically significant (at least with $90 \%$ confidence). In addition, although the EMA-TS performs better than EMA considering the objective function value, due to its relatively high computational effort, the MIC of this hybrid is not higher than that of EMA.

The results presented in Table 8 show that the hybrid of EMA and VNS outperforms other algorithms in medium and large instances (the most interesting) in terms of solution quality. Given the timeframe of the SCND problem, the execution time is not a concern; therefore, the EMA-VNS hybrid is preferable in our case; however, if the target would be a real-time application, then the EMA-SA hybrid may be considered as the superior algorithm.

\section{Conclusion and Future Works}

In this paper, the designing of a multiproduct multimode multiperiod supply chain network considering traffic congestion and both supply-side and demand-side uncertainties is 
TABLE 12: The data for links free flow travel times.

\begin{tabular}{lccccccc}
\hline \multirow{2}{*}{ Free flow travel time } & \multicolumn{2}{c}{ City 1 } & \multicolumn{2}{c}{ City 2 } & \multicolumn{2}{c}{ City 3 } & \multicolumn{2}{c}{ City 4 } \\
& Period 1 & Period 2 & Period 1 & Period 2 & Period 1 & Period 2 & Period 1 \\
\hline City 1 & 1 & 8 & 9 & 9 & 3 & 3 & 2 \\
City 2 & 1 & 9 & 1 & 6 & 9 & 2 & 9 \\
City 3 & 9 & 6 & 7 & 9 & 2 & 5 & 6 \\
City 4 & 4 & 8 & 6 & 6 & 7 & 7 & 9 \\
\hline
\end{tabular}

TABLE 13: The data for transportation costs.

\begin{tabular}{llcccccccc}
\hline \multirow{2}{*}{ Transportation costs } & \multicolumn{2}{c}{ City 1 } & \multicolumn{2}{c}{ City 2 } & \multicolumn{2}{c}{ City 3 } & \multicolumn{2}{c}{ City 4 } \\
& & Period 1 & Period 2 & Period 1 & Period 2 & Period 1 & Period 2 & Period 1 & Period 2 \\
\hline \multirow{2}{*}{ City 1 } & Mode 1 & 0.044 & 0.705 & 0.971 & 0.744 & 0.857 & 0.492 & 0.118 & 0.429 \\
& Mode 2 & 0.796 & 0.434 & 0.962 & 0.714 & 0.877 & 0.297 & 0.11 & 0.456 \\
\hline \multirow{2}{*}{ City 2 } & Mode 1 & 0.526 & 0.166 & 0.997 & 0.152 & 0.692 & 0.744 & 0.066 & 0.351 \\
& Mode 2 & 0.106 & 0.745 & 0.987 & 0.893 & 0.129 & 0.496 & 0.501 & 0.399 \\
\hline \multirow{2}{*}{ City 3 } & Mode 1 & 0.176 & 0.486 & 0.376 & 0.356 & 0.058 & 0.434 & 0.402 & 0.013 \\
& Mode 2 & 0.687 & 0.132 & 0.895 & 0.128 & 0.274 & 0.3 & 0.109 & 0.131 \\
\hline \multirow{2}{*}{ City 4 } & Mode 1 & 0.694 & 0.751 & 0.602 & 0.285 & 0.916 & 0.048 & 0.273 & 0.442 \\
& Mode 2 & 0.568 & 0.022 & 0.346 & 0.564 & 0.139 & 0.381 & 0.189 & 0.61 \\
\hline
\end{tabular}

TABLE 14: The data for facility costs.

\begin{tabular}{|c|c|c|c|c|c|c|}
\hline \multirow{2}{*}{ Facility costs } & \multicolumn{2}{|c|}{ Maintenance cost } & \multicolumn{2}{|c|}{ Opening cost } & \multicolumn{2}{|c|}{ Closing cost } \\
\hline & Period 1 & Period 2 & Period 1 & Period 2 & Period 1 & Period 2 \\
\hline City 1 & 0.405 & 3.883 & 80.098 & 77.651 & 0.810 & 7.765 \\
\hline City 2 & 2.169 & 4.858 & 43.383 & 97.153 & 4.338 & 9.715 \\
\hline City 3 & 0.382 & 0.837 & 57.640 & 36.748 & 0.764 & 1.675 \\
\hline City 4 & 2.982 & 1.404 & 59.642 & 28.082 & 5.964 & 2.808 \\
\hline
\end{tabular}

investigated and an optimization model is proposed. In order to justify the proposed model, a small numerical problem is solved by LINGO. The experiments with the numerical example show that neglecting the congestion cost in SCND results in preferring the cheaper but more congested routes for transportation of products, and/or cheaper transportation vehicles that have higher congestion costs are given preference. This fact shows the significance of considering traffic congestion in SCND.

In addition, to tackle the complexity of the larger problems, two hybrid Electromagnetism-Like Algorithms are presented and several test problems are solved. The numerical experiments results show that proposed algorithms are promising.

The outcomes of the experiment with the small test problem yield the applicability of the proposed model in many real-world situations. Therefore, one of future studies may be comprehensive real case study and sensitivity analyses of an application of the proposed model. Uncertainty may exist in other parameters such as transportation costs. Therefore, a possible future research may be the study of uncertainty in parameters other than those considered in this research.
TABLE 15: The data for products demands.

\begin{tabular}{lcccc}
\hline \multirow{2}{*}{ Demand } & \multicolumn{2}{c}{ Product 1 } & \multicolumn{2}{c}{ Product 2 } \\
& Period 1 & Period 2 & Period 1 & Period 2 \\
\hline City 1 & 3 & 2 & 2 & 1 \\
City 2 & 2 & 1 & 1 & 3 \\
City 3 & 3 & 2 & 2 & 2 \\
City 4 & 2 & 3 & 2 & 1 \\
\hline
\end{tabular}

\section{Appendix}

The data for the small test problem are presented here. The traffic capacity, transportation costs, link flows, and so forth, from a city to itself, can be interpreted as the local transportation properties such as total capacity of urban roads within the city. Similar discussion can be presented regarding the transportation costs, basic link flow, and free flow travel times.

It should be noted that the aforementioned data are generated randomly. In addition, the monetary value of time and the interest rate are assumed to be constant for both 
TABLE 16: The data for products potential production capacities.

\begin{tabular}{lcccc}
\hline \multirow{2}{*}{ Demand } & \multicolumn{2}{c}{ Product 1 } & \multicolumn{2}{c}{ Product 2 } \\
& Period 1 & Period 2 & Period 1 & Period 2 \\
\hline City 1 & 8 & 10 & 6 & 6 \\
City 2 & 8 & 8 & 8 & 6 \\
City 3 & 6 & 8 & 6 & 8 \\
City 4 & 6 & 6 & 8 & 6 \\
\hline
\end{tabular}

periods and equal 0.01 and 0.1 , respectively. $\alpha$ and $\beta$ are set to 0.15 and 4, respectively. Furthermore, all violation cost coefficients for demand and supply are set to 1 and the spreads for all fuzzy numbers are set to 0.1 . The data are summarized in Tables 9, 10, 11, 12, 13, 14, 15, and 16.

\section{Conflict of Interests}

The authors declare that there is no conflict of interests.

\section{References}

[1] R. K. Oliver and M. D. Webber, "Supply-chain management: logistics catches up with strategy," Outlook, vol. 5, no. 1, pp. 4247, 1982.

[2] W. Chandraprakaikul, T. S. Baines, and R. Y. Lim, "Strategic positioning of manufacturing operations within global supply chains," Proceedings of the Institution of Mechanical Engineers, Part B: Journal of Engineering Manufacture, vol. 224, no. 5, pp. 831-846, 2010.

[3] H. Davarzani and S. Rezapour, "Supply network design," in Supply Chain and Logistics in National, International and Governmental Environment, R. Z. Farahani, N. Asgari, and H. Davarzani, Eds., Contributions to Management Science, pp. 105-128, Physica, Heidelberg, Germany, 2009.

[4] D. Simchi-Levi, P. Kaminsky, and E. Simchi-Levi, Designing and Managing the Supply Chain: Concepts, Strategies, and Case Sudies, McGraw-Hill, Irwin, Boston, Mass, USA, 2003.

[5] M. S. Pishvaee, J. Razmi, and S. A. Torabi, "An accelerated Benders decomposition algorithm for sustainable supply chain network design under uncertainty: a case study of medical needle and syringe supply chain," Transportation Research Part E: Logistics and Transportation Review, vol. 67, pp. 14-38, 2014.

[6] C. R. Carter and D. S. Rogers, "A framework of sustainable supply chain management: moving toward new theory," International Journal of Physical Distribution \& Logistics Management, vol. 38 , no. 5, pp. 360-387, 2008.

[7] J. Jouzdani, S. J. Sadjadi, and M. Fathian, "Dynamic dairy facility location and supply chain planning under traffic congestion and demand uncertainty: a case study of Tehran," Applied Mathematical Modelling, vol. 37, no. 18-19, pp. 8467-8483, 2013.

[8] K. Subulan, A. S. Taşan, and A. Baykasoğlu, "A fuzzy goal programming model to strategic planning problem of a lead/acid battery closed-loop supply chain," Journal of Manufacturing Systems, 2014.

[9] S. J. Sadjadi, R. Soltani, and A. Eskandarpour, "Location based treatment activities for end of life products network design under uncertainty by a robust multi-objective memetic-based heuristic approach," Applied Soft Computing, vol. 23, pp. 215226, 2014.
[10] A. Osmani and J. Zhang, "Economic and environmental optimization of a large scale sustainable dual feedstock lignocellulosic-based bioethanol supply chain in a stochastic environment," Applied Energy, vol. 114, pp. 572-587, 2014.

[11] A. Hasani, S. H. Zegordi, and E. Nikbakhsh, "Robust closedloop global supply chain network design under uncertainty: the case of the medical device industry," International Journal of Production Research, vol. 53, no. 5, pp. 1596-1624, 2014.

[12] R. Dubey and A. Gunasekaran, "Sustainable supply chain network design: a case of Indian company," International Journal of Logistics Research and Applications, pp. 1-21, 2014.

[13] T. Boukherroub, A. Ruiz, A. Guinet, and J. Fondrevelle, "An integrated approach for sustainable supply chain planning," Computers \& Operations Research, vol. 54, pp. 180-194, 2015.

[14] M. Brandenburg, "Low carbon supply chain configuration for a new product-a goal programming approach," International Journal of Production Research, pp. 1-23, 2015.

[15] K. Govindan, A. Jafarian, and V. Nourbakhsh, "Bi-objective integrating sustainable order allocation and sustainable supply chain network strategic design with stochastic demand using a novel robust hybrid multi-objective metaheuristic," Computers \& Operations Research, 2015.

[16] M. Eskandarpour, P. Dejax, J. Miemczyk, and O. Péton, "Sustainable supply chain network design: an optimization-oriented review," Omega, vol. 54, pp. 11-32, 2015.

[17] M. S. Roni, S. D. Eksioglu, E. Searcy, and K. Jha, "A supply chain network design model for biomass co-firing in coal-fired power plants," Transportation Research Part E: Logistics and Transportation Review, vol. 61, pp. 115-134, 2014.

[18] W. Klibi, A. Martel, and A. Guitouni, "The design of robust value-creating supply chain networks: a critical review," European Journal of Operational Research, vol. 203, no. 2, pp. 283293, 2010.

[19] M. C. Georgiadis, P. Tsiakis, P. Longinidis, and M. K. Sofioglou, "Optimal design of supply chain networks under uncertain transient demand variations," Omega, vol. 39, no. 3, pp. 254-272, 2011.

[20] K. J. Mizgier, S. M. Wagner, and J. A. Holyst, "Modeling defaults of companies in multi-stage supply chain networks," International Journal of Production Economics, vol. 135, no. 1, pp. 14-23, 2012.

[21] S. R. Cardoso, A. P. Barbosa-Póvoa, and S. Relvas, "Design and planning of supply chains with integration of reverse logistics activities under demand uncertainty," European Journal of Operational Research, vol. 226, no. 3, pp. 436-451, 2013.

[22] G. Yang and Y. Liu, "Designing fuzzy supply chain network problem by mean-risk optimization method," Journal of Intelligent Manufacturing, vol. 26, no. 3, pp. 447-458, 2015.

[23] M. S. Pishvaee and S. A. Torabi, "A possibilistic programming approach for closed-loop supply chain network design under uncertainty," Fuzzy Sets and Systems, vol. 161, no. 20, pp. 26682683, 2010.

[24] J. Jouzdani and M. Fathian, "A robust mathematical model for route planning of a third-party logistics provider," in Proceedings of the 42nd International Conference on Computers and Industrial Engineering (CIE '12), pp. 599-608, Cape Town International Convention Centre (CTICC), Cape Town, South Africa, July 2012.

[25] M. Ramezani, M. Bashiri, and R. Tavakkoli-Moghaddam, "A robust design for a closed-loop supply chain network under an uncertain environment," The International Journal of Advanced Manufacturing Technology, vol. 66, no. 5-8, pp. 825-843, 2013. 
[26] W. Klibi and A. Martel, "The design of robust value-creating supply chain networks," OR Spectrum. Quantitative Approaches in Management, vol. 35, no. 4, pp. 867-903, 2013.

[27] J. Jouzdani and M. Fathian, "A linear MmTSP formulation of robust location-routing problem: a dairy products supply chain case study," International Journal of Applied Decision Sciences, vol. 7, no. 3, p. 327, 2014.

[28] A. Jabbarzadeh, S. G. Jalali Naini, H. Davoudpour, and N. Azad, "Designing a supply chain network under the risk of disruptions," Mathematical Problems in Engineering, vol. 2012, Article ID 234324, 23 pages, 2012.

[29] D. Peidro, J. Mula, R. Poler, and F.-C. Lario, "Quantitative models for supply chain planning under uncertainty: a review," International Journal of Advanced Manufacturing Technology, vol. 43, no. 3-4, pp. 400-420, 2009.

[30] G. L. Nemhauser and L. A. Wolsey, Integer and Combinatorial Optimization, vol. 18 of Wiley-Interscience Series in Discrete Mathematics and Optimization, John Wiley \& Sons, New York, NY, USA, 1988.

[31] H. Soleimani and G. Kannan, "A hybrid particle swarm optimization and genetic algorithm for closed-loop supply chain network design in large-scale networks," Applied Mathematical Modelling, 2014.

[32] S. M. Mousavi, A. Bahreininejad, S. N. Musa, and F. Yusof, "A modified particle swarm optimization for solving the integrated location and inventory control problems in a two-echelon supply chain network," Journal of Intelligent Manufacturing.

[33] E. Roghanian and P. Pazhoheshfar, "An optimization model for reverse logistics network under stochastic environment by using genetic algorithm," Journal of Manufacturing Systems, vol. 33, no. 3, pp. 348-356, 2014.

[34] Y. Cardona-Valdés, A. Álvarez, and J. Pacheco, "Metaheuristic procedure for a bi-objective supply chain design problem with uncertainty," Transportation Research, Part B: Methodological, vol. 60, pp. 66-84, 2014.

[35] S. H. R. Pasandideh, S. T. A. Niaki, and K. Asadi, "Bi-objective optimization of a multi-product multi-period three-echelon supply chain problem under uncertain environments: NSGAII and NRGA," Information Sciences, vol. 292, pp. 57-74, 2015.

[36] S. I. Birbil and S.-C. Fang, "An electromagnetism-like mechanism for global optimization," Journal of Global Optimization, vol. 25, no. 3, pp. 263-282, 2003.

[37] Z. Naji-Azimi, P. Toth, and L. Galli, "An electromagnetism metaheuristic for the unicost set covering problem," European Journal of Operational Research, vol. 205, no. 2, pp. 290-300, 2010.

[38] P.-C. Chang, S.-H. Chen, and C.-Y. Fan, "A hybrid electromagnetism-like algorithm for single machine scheduling problem," Expert Systems with Applications, vol. 36, no. 2, pp. 12591267, 2009.

[39] H. Davoudpour and M. Hadji Molana, "Solving flow shop sequencing problem for deteriorating jobs by using electro magnetic algorithm," Journal of Applied Sciences, vol. 8, no. 22, pp. 4121-4128, 2008.

[40] B. Naderi, R. Tavakkoli-Moghaddam, and M. Khalili, "Electromagnetism-like mechanism and simulated annealing algorithms for flowshop scheduling problems minimizing the total weighted tardiness and makespan," Knowledge-Based Systems, vol. 23, no. 2, pp. 77-85, 2010.

[41] D. Debels, B. de Reyck, R. Leus, and M. Vanhoucke, "A hybrid scatter search/electromagnetism meta-heuristic for project scheduling," European Journal of Operational Research, vol. 169, no. 2, pp. 638-653, 2006.

[42] A. Jamili, M. A. Shafia, and R. Tavakkoli-Moghaddam, "A hybridization of simulated annealing and electromagnetismlike mechanism for a periodic job shop scheduling problem," Expert Systems with Applications, vol. 38, no. 5, pp. 5895-5901, 2011.

[43] P. Wu, K.-J. Yang, and H.-C. Fang, "A revised EM-like algorithm + K-OPT method for solving the traveling salesman problem," in Proceedings of the 1st International Conference on Innovative Computing, Information and Control (ICICIC '06), pp. 546-549, Beijing, China, September 2006.

[44] N. Javadian, M. G. Alikhani, and R. Tavakkoli-Moghaddam, "A discrete binary version of the electromagnetism-like heuristic for solving traveling salesman problem," in Advanced Intelligent Computing Theories and Applications. With Aspects of Artificial Intelligence, vol. 5227 of Lecture Notes in Computer Science, pp. 123-130, Springer, Berlin, Germany, 2008.

[45] F. Jolai, R. Tavakkoli-Moghaddam, A. Golmohammadi, and B. Javadi, "An Electromagnetism-like algorithm for cell formation and layout problem," Expert Systems with Applications, vol. 39, no. 2, pp. 2172-2182, 2012.

[46] P. Doganis and H. Sarimveis, "Optimal scheduling in a yogurt production line based on mixed integer linear programming," Journal of Food Engineering, vol. 80, no. 2, pp. 445-453, 2007.

[47] V. de Rosa, M. Gebhard, E. Hartmann, and J. Wollenweber, "Robust sustainable bi-directional logistics network design under uncertainty," International Journal of Production Economics, vol. 145, no. 1, pp. 184-198, 2013.

[48] J. Jouzdani, F. Barzinpour, and M. Fathian, "An improved electromagnetism-like algorithm for global optimization," in Proceedings of the 42nd International Conference on Computers and Industrial Engineering (CIE '12), pp. 879-888, Cape Town, South Africa, July 2012.

[49] S. Kirkpatrick, C. D. Gelatt Jr., and M. P. Vecchi, "Optimization by simulated annealing," Science, vol. 220, no. 4598, pp. 671-680, 1983.

[50] P. Subramanian, N. Ramkumar, T. T. Narendran, and K. Ganesh, "PRISM: PRIority based SiMulated annealing for a closed loop supply chain network design problem," Applied Soft Computing Journal, vol. 13, no. 2, pp. 1121-1135, 2013.

[51] E. Urra, C. Cubillos, and D. Cabrera-Paniagua, "A hyperheuristic for the dial-a-ride problem with time windows," Mathematical Problems in Engineering, vol. 2015, Article ID 707056, 12 pages, 2015.

[52] D. Chen, S. Zhou, Y. Xie, and X. Li, "Optimal facility location model based on genetic simulated annealing algorithm for siting urban refueling stations," Mathematical Problems in Engineering. In press.

[53] İ. Küçükoğlu and N. Öztürk, "An advanced hybrid metaheuristic algorithm for the vehicle routing problem with backhauls and time windows," Computers \& Industrial Engineering, 2014.

[54] A. Salehipour, M. Modarres, and L. M. Naeni, "An efficient hybrid meta-heuristic for aircraft landing problem," Computers \& Operations Research, vol. 40, no. 1, pp. 207-213, 2013.

[55] S. M. Mousavi and R. Tavakkoli-Moghaddam, "A hybrid simulated annealing algorithm for location and routing scheduling problems with cross-docking in the supply chain," Journal of Manufacturing Systems, vol. 32, no. 2, pp. 335-347, 2013. 
[56] N. Mladenović and P. Hansen, "Variable neighborhood search," Computers \& Operations Research, vol. 24, no. 11, pp. 1097-1100, 1997.

[57] J. Behnamian, M. Zandieh, and S. M. T. Fatemi Ghomi, "Parallel-machine scheduling problems with sequence-dependent setup times using an ACO, SA and VNS hybrid algorithm," Expert Systems with Applications, vol. 36, no. 6, pp. 9637-9644, 2009.

[58] Y. Wen, H. Xu, and J. Yang, "A heuristic-based hybrid geneticvariable neighborhood search algorithm for task scheduling in heterogeneous multiprocessor system," Information Sciences, vol. 181, no. 3, pp. 567-581, 2011.

[59] A. Salehipour, K. Sörensen, P. Goos, and O. Bräysy, "Efficient GRASP+VND and GRASP+VNS metaheuristics for the traveling repairman problem," 4OR, vol. 9, no. 2, pp. 189-209, 2011.

[60] M. M. Paydar and M. Saidi-Mehrabad, "A hybrid geneticvariable neighborhood search algorithm for the cell formation problem based on grouping efficacy," Computers \& Operations Research, vol. 40, no. 4, pp. 980-990, 2013.

[61] M. Madani-Isfahani, R. Tavakkoli-Moghaddam, and B. Naderi, "Multiple cross-docks scheduling using two meta-heuristic algorithms," Computers \& Industrial Engineering, vol. 74, pp. 129-138, 2014.

[62] Z. Miao, K. Fu, and F. Yang, "A hybrid genetic algorithm for the multiple crossdocks problem," Mathematical Problems in Engineering, vol. 2012, Article ID 316908, 18 pages, 2012.

[63] N. van Hop, "Fuzzy stochastic goal programming problems," European Journal of Operational Research, vol. 176, no. 1, pp. 7786, 2007.

[64] S. G. J. Naini, M. M. Paydar, J. Jouzdani, and M. Fathian, "Fuzzy stochastic linear programming-based approach for multiple departures single destination multiple travelling salesman problem," International Journal of Operational Research, vol. 17, no. 4, pp. 417-435, 2013.

[65] K. Liu, Y. Zhou, and Z. Zhang, "Capacitated location model with online demand pooling in a multi-channel supply chain," European Journal of Operational Research, vol. 207, no. 1, pp. 218-231, 2010.

[66] A. A. Rentizelas and I. P. Tatsiopoulos, "Locating a bioenergy facility using a hybrid optimization method," International Journal of Production Economics, vol. 123, no. 1, pp. 196-209, 2010.

[67] M. I. G. Salema, A. P. Barbosa-Povoa, and A. Q. Novais, "Simultaneous design and planning of supply chains with reverse flows: a generic modelling framework," European Journal of Operational Research, vol. 203, no. 2, pp. 336-349, 2010.

[68] T. P. N. Le and T.-R. Lee, "Model selection with considering the $\mathrm{CO}_{2}$ emission alone the global supply chain," Journal of Intelligent Manufacturing, vol. 24, no. 4, pp. 653-672, 2013.

[69] Y. Bai, T. Hwang, S. Kang, and Y. Ouyang, "Biofuel refinery location and supply chain planning under traffic congestion," Transportation Research Part B: Methodological, vol. 45, no. 1, pp. 162-175, 2011.

[70] S. M. J. Mirzapour Al-e-Hashem, H. Malekly, and M. B. Aryanezhad, "A multi-objective robust optimization model for multi-product multi-site aggregate production planning in a supply chain under uncertainty," International Journal of Production Economics, vol. 134, no. 1, pp. 28-42, 2011.

[71] M. S. Pishvaee, M. Rabbani, and S. A. Torabi, "A robust optimization approach to closed-loop supply chain network design under uncertainty," Applied Mathematical Modelling, vol. 35, no. 2, pp. 637-649, 2011.
[72] C.-W. Chen and Y. Fan, "Bioethanol supply chain system planning under supply and demand uncertainties," Transportation Research Part E: Logistics and Transportation Review, vol. 48, no. 1, pp. 150-164, 2012.

[73] F. Wang, X. Lai, and N. Shi, "A multi-objective optimization for green supply chain network design," Decision Support Systems, vol. 51, no. 2, pp. 262-269, 2011.

[74] A. M. Kostin, G. Guillén-Gosálbez, F. D. Mele, M. J. Bagajewicz, and L. Jiménez, "Design and planning of infrastructures for bioethanol and sugar production under demand uncertainty," Chemical Engineering Research and Design, vol. 90, no. 3, pp. 359-376, 2012.

[75] A. Almansoori and N. Shah, "Design and operation of a stochastic hydrogen supply chain network under demand uncertainty," International Journal of Hydrogen Energy, vol. 37, no. 5, pp. 3965-3977, 2012.

[76] M. Bashiri, H. Badri, and J. Talebi, "A new approach to tactical and strategic planning in production-distribution networks," Applied Mathematical Modelling, vol. 36, no. 4, pp. 1703-1717, 2012.

[77] S. Wang and J. Watada, "A hybrid modified PSO approach to VaR-based facility location problems with variable capacity in fuzzy random uncertainty," Information Sciences, vol. 192, pp. 3-18, 2012.

[78] H. Sadjady and H. Davoudpour, "Two-echelon, multi-commodity supply chain network design with mode selection, leadtimes and inventory costs," Computers \& Operations Research, vol. 39, no. 7, pp. 1345-1354, 2012.

[79] B. Vahdani, R. Tavakkoli-Moghaddam, F. Jolai, and A. Baboli, "Reliable design of a closed loop supply chain network under uncertainty: an interval fuzzy possibilistic chance-constrained model," Engineering Optimization, vol. 45, no. 6, pp. 745-765, 2013.

[80] A. Baghalian, S. Rezapour, and R. Z. Farahani, "Robust supply chain network design with service level against disruptions and demand uncertainties: a real-life case," European Journal of Operational Research, vol. 227, no. 1, pp. 199-215, 2013.

[81] S. M. Hatefi and F. Jolai, "Robust and reliable forward-reverse logistics network design under demand uncertainty and facility disruptions," Applied Mathematical Modelling, vol. 38, no. 9-10, pp. 2630-2647, 2014.

[82] A. Jindal and K. S. Sangwan, "Closed loop supply chain network design and optimisation using fuzzy mixed integer linear programming model," International Journal of Production Research, vol. 52, no. 14, pp. 4156-4173, 2013.

[83] H. Soleimani, M. Seyyed-Esfahani, and M. A. Shirazi, "Designing and planning a multi-echelon multi-period multi-product closed-loop supply chain utilizing genetic algorithm," The International Journal of Advanced Manufacturing Technology, vol. 68, no. 1-4, pp. 917-931, 2013.

[84] A. Jabbarzadeh, B. Fahimnia, and S. Seuring, "Dynamic supply chain network design for the supply of blood in disasters: a robust model with real world application," Transportation Research Part E: Logistics and Transportation Review, vol. 70, pp. 225-244, 2014.

[85] N. Shabani, T. Sowlati, M. Ouhimmou, and M. Rönnqvist, "Tactical supply chain planning for a forest biomass power plant under supply uncertainty," Energy, vol. 78, pp. 346-355, 2014.

[86] R. S. Elliott, Electromagnetics: History, Theory, and Applications, IEEE Press, 1999.

[87] C.-T. Tseng and K.-H. Chen, "An electromagnetism-like mechanism for the single machine total stepwise tardiness problem 
with release dates," Engineering Optimization, vol. 45, no. 12, pp. 1431-1448, 2013.

[88] M. Khalili and R. Tavakkoli-Moghaddam, "A multi-objective electromagnetism algorithm for a bi-objective flowshop scheduling problem," Journal of Manufacturing Systems, vol. 31, no. 2, pp. 232-239, 2012.

[89] J. Jouzdani, F. Barzinpour, M. A. Shafia, and M. Fathian, "Applying simulated annealing to a generalized cell formation problem considering alternative routings and machine reliability," AsiaPacific Journal of Operational Research, vol. 31, no. 4, Article ID 1450021, 2014.

[90] I. B. Osman, "Metaheuristics: models, design and analysis," in Proceedings of the 5th Asia Pacific Industrial Engineering and Management Systems Conference, Asia Pacific Industrial Engineering and Management Society, Gold Coast, Australia, 2004. 


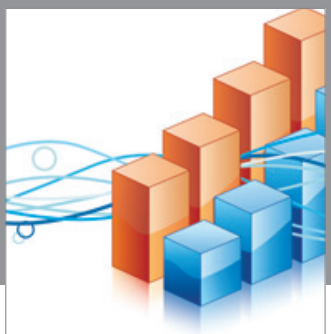

Advances in

Operations Research

vatem alat4

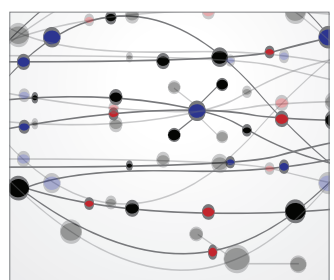

\section{The Scientific} World Journal
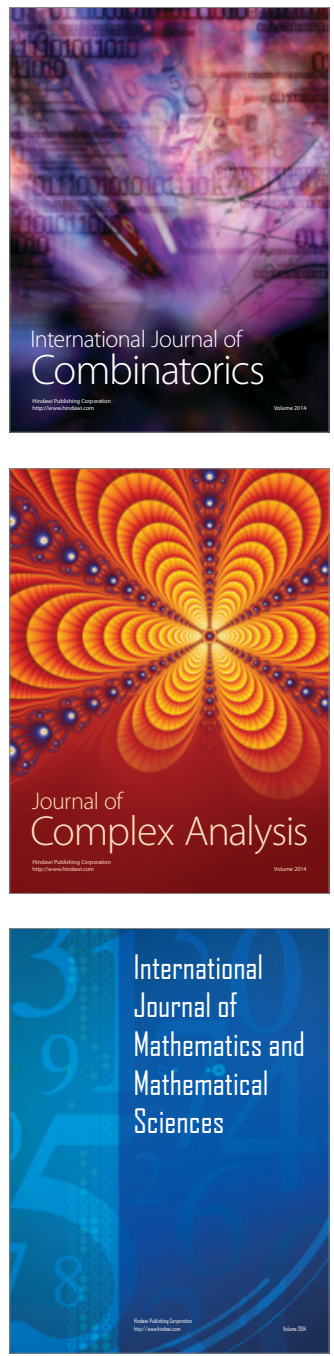
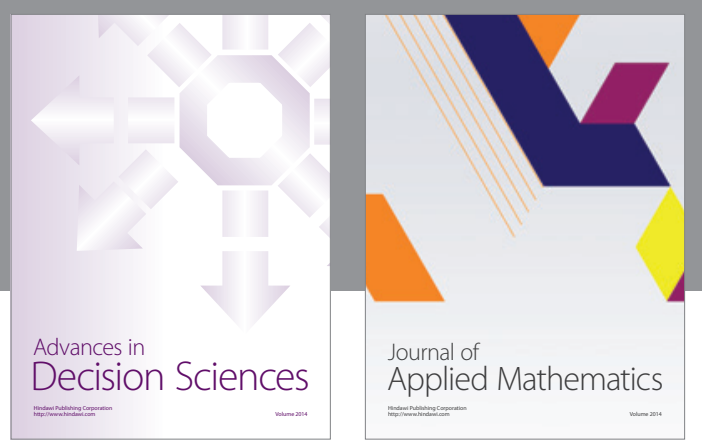

Algebra

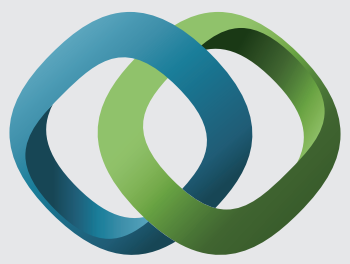

\section{Hindawi}

Submit your manuscripts at

http://www.hindawi.com
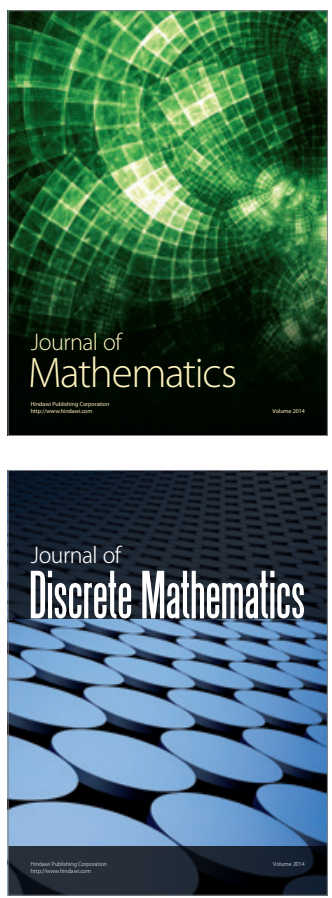

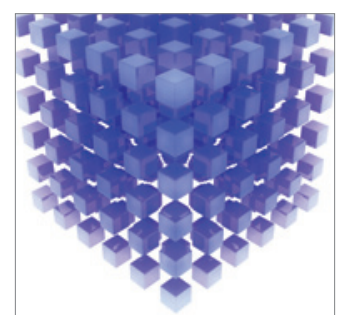

Mathematical Problems in Engineering
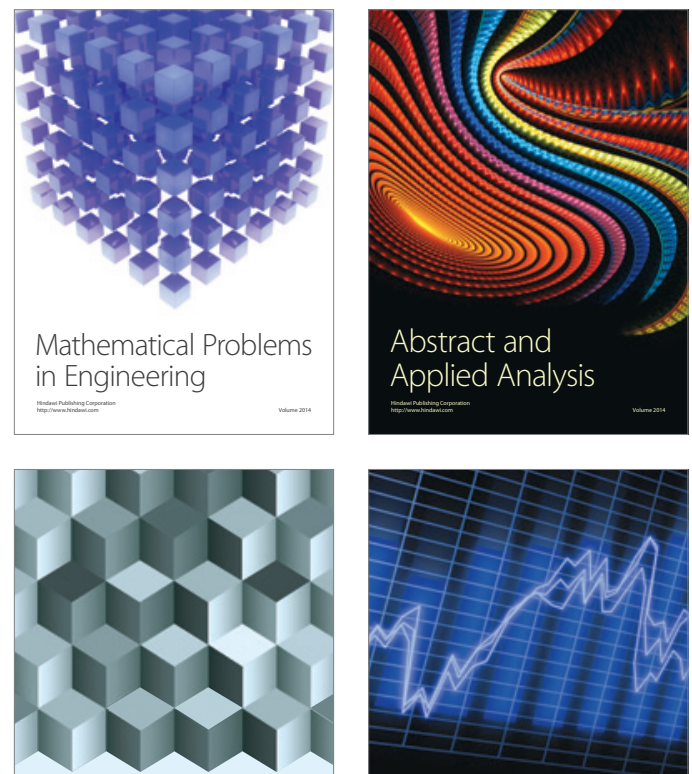

Journal of

Function Spaces

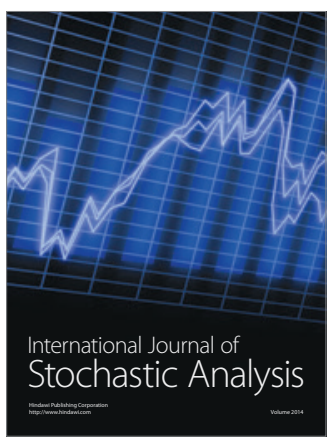

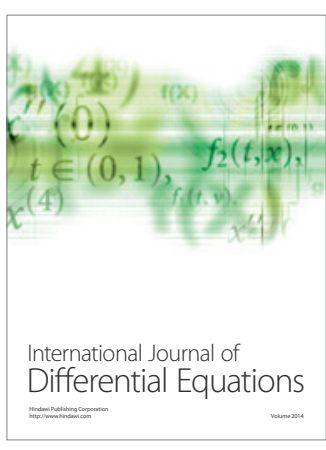
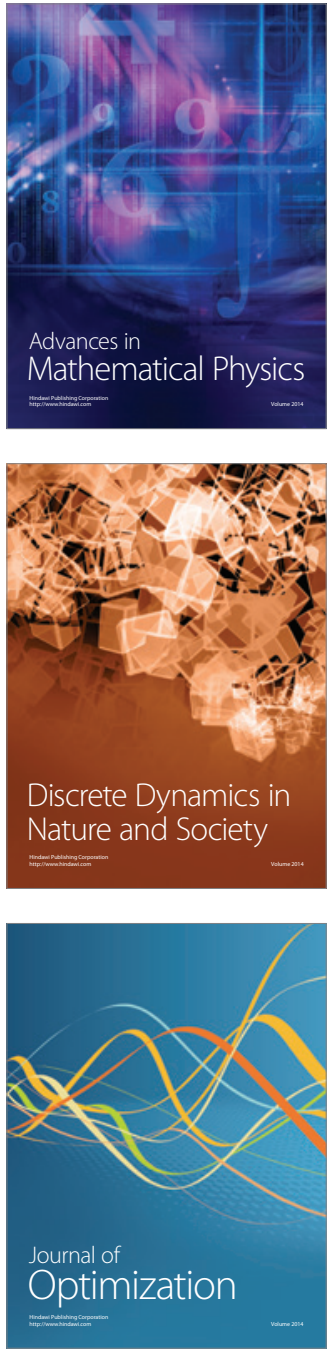